\title{
Finite element computation of grating scattering matrices and application to photonic crystal band calculations
}

\author{
Kokou Dossou*, Michael A. Byrne and Lindsay C. Botten \\ Centre for Ultrahigh-Bandwidth Devices for Optical Systems and Department of \\ Mathematical Sciences; University of Technology, Sydney; PO Box 123; Broadway, \\ New South Wales 2007, Australia
}

\begin{abstract}
We consider the calculation of the band structure and Bloch mode basis of twodimensional photonic crystals, modelled as stacks of one-dimensional diffraction gratings. The scattering properties of each grating are calculated using an efficient finite element method (FEM) and allow the complete mode structure to be derived from a transfer matrix method. A range of numerical examples showing the accuracy, flexibility and utility of the method is presented.
\end{abstract}

Key words: Finite element method; Photonic crystals; Bloch modes; scattering matrix; transfer matrix method.

\section{Introduction}

Over the past decade, photonic crystals (PC) have emerged as one of the most active areas of contemporary optics research, due largely to their unique intrinsic properties which allow for substantial control over the flow of light and which, in turn, open up the potential for miniaturised devices embedded in compact, integrated optical circuits. In such "photonic chips", different components may be connected using complicated "wiring" networks comprising waveguides with bends [41], $Y$ - [4] and $T$-junctions [20], channel drop filters [21], couplers [5,42], superprisms [28], Mach-Zehnder interferometers [32]) and so on. The modelling of such structures is theoretically and computationally

* Corresponding author. Tel.: 61-2-9514 2145, fax: 61-2-9514 2260

Email address: Kokou.Dossou@uts.edu.au (Kokou Dossou). 
challenging because of their geometrical complexity, the strong scattering environment associated with both the wavelength scale of the structure and the (often) high index contrast of the constituent materials. Accordingly, the development of efficient, accurate and robust numerical tools is an important aspect of photonic crystal research.

In this paper, we consider two-dimensional rod-type and hole-type photonic crystals, with the analysis that is presented being easily extended to handle $2.5 \mathrm{D}$ systems (in which fields are three-dimensional in nature, but the geometry is two dimensional, as in an optical fibre). The mathematical formulation is based on the theory of Bloch modes and the solution of eigenvalue problems. For problems modelled in the frequency domain, there are two basic solution approaches depending on how the unknown eigenvalue parameter is chosen. Either the Bloch wave vector $\mathbf{k}_{0}$ can be fixed, in which case the frequency $\omega$ is the eigenvalue, or alternatively, $\omega$ and one (or two, in the case of $3 \mathrm{D}$ photonic crystals) components of $\mathbf{k}_{0}$ can be chosen, with the other remaining Bloch vector component becoming the unknown eigenvalue. The analysis of the Bloch modes when the Bloch wave vector $\mathbf{k}_{0}$ is given and the frequency $\omega$ is unknown leads to Hermitian eigenproblems which can be efficiently solved using various existing computational methods, such as plane wave expansion techniques $[26,27]$ and finite element methods [2,15]. Such an approach, however, becomes difficult to use for dispersive media, in which the dielectric constant depends on the frequency (which is the eigenvalue). In contrast, methods which first fix the frequency (e.g., [34]) can easily handle dispersive medium problems and can also be used to efficiently analyse many other physically relevant applications where the frequency is given.

In this paper we concern ourselves with the spectral problem where the values of the frequency $\omega$ and one of the two components of $\mathbf{k}_{0}=\left(\alpha_{0}, \beta_{0}\right), \alpha_{0}$ for instance, are fixed. However, the resulting eigenvalue problem is nonlinear and even for lossless media and real values of $\alpha_{0}$, complex valued eigenvalues $\beta_{0}$ can occur, corresponding to the evanescent modes. Because of the nonlinearity, the direct application of standard numerical solution techniques for eigenproblems is difficult and computationally expensive. These problems can be circumvented, however, by using the transfer matrix formalism $[34,7]$ to revert the computational solution to one of a standard algebraic eigenvalue problem. In this approach, an infinite two-dimensional photonic crystal is modelled as a periodic stack of grating layers. The transfer matrix relates the fields above and below the grating, and by applying the Bloch condition, the eigenvalues of the transfer matrix appear in the form of exponentials involving the components of the Bloch vector.

The transfer matrix is typically a dense matrix of low dimension and the numerical calculation of its eigenvalues is computationally inexpensive, although the construction of the transfer matrix itself may be computationally 
expensive. In order to benefit from the numerous techniques available in the literature on scattering by diffraction gratings [35], it is useful to formulate the transfer matrix eigenproblem using grating scattering matrices $[7,23,39]$ which relate the output (reflected and transmitted) plane wave amplitudes to the incident field amplitudes.

Scattering matrices may be generated using a variety of techniques and, indeed, our group has extensive experience in the use of the multipole method [8]. While the multipole method has proven to be an effective tool for modelling PC structures composed of cylinder gratings, it has a number of limitations, the most obvious of which is the restriction on the range of geometries that can be accommodated. This method also has limitations when dealing with cylinder gratings that overlap or interpenetrate to any significant extent. While a hybrid multipole-plane wave method [8] can handle modest interpenetration of cylinder layers, the method breaks down when the interpenetration exceeds some threshold, a phenomenon which is directly related to the Rayleigh controversy (see, for example, Ref [35, chapter 1]) concerning the validity of plane wave expansions for representing outgoing fields within the grooves of diffraction gratings. The combination of these issues has thus led us to develop a general technique based on the finite element method - one that is able to accommodate a wide class of geometries and which overcomes the shortcomings of the multipole method. Accordingly, this paper outlines a finite element approach to generating scattering matrices and goes on to explore the use of this in the context of the transfer matrix method and applications that are of interest in the study of photonic crystal devices.

Since construction of the scattering matrices involves the solution of a grating diffraction problem (through the solution of Maxwell's equations) for each diffraction order used in the plane wave (Rayleigh) expansion, their calculation dominates the computational effort involved in calculating the Bloch modes of the structure. As we will see, however, the process is made efficient by observing that the solution of each of these diffraction problems (which generates a particular column of the scattering matrix) involves the calculation and LU factorisation of only one finite element matrix. By taking into account the particular structure of the discretised system, we also significantly reduce the computational cost of building the right hand side of the final matrix system. Thus, the scattering matrices can be calculated reasonably quickly.

In what follows, we contextualise the scattering matrices by outlining the transfer matrix method for generating the Bloch modes of the photonic crystal. We then proceed to develop the weak formulation of the problem in Sec. 3 and describe the FEM discretisation of the variational problem in Sec. 4. In Sec. 5, we apply these tools, considering the convergence properties of the FEM, the symmetry properties of the eigenvalue distribution, and the photonic crystal band diagrams. We also propose an approach to treat a photonic crystal with 
a perfectly conducting metallic inclusions when the grating layer interfaces intersect the inclusions. Finally to illustrate how our approach can be applied to a finite size photonic crystal, we analyse the optimisation of taper shapes for efficient coupling of light from a photonic crystal waveguide to free space (with details of the algorithm given in Appendix A).

\section{The eigenvalue problem}

We assume that the dielectric constant $\varepsilon$ is invariant with respect to the variable $z$ (the axis of the grating inclusions) and is periodic in the $(x, y)$-plane with respect to a lattice $[1,37]$

$$
\mathcal{L}=\left\{m \mathbf{e}_{1}+n \mathbf{e}_{2}, m, n \in \mathbb{Z}\right\}
$$

where $\mathbf{e}_{1} \in \mathbb{R}^{2}$ and $\mathbf{e}_{2} \in \mathbb{R}^{2}$ are the basis vectors of the lattice. The reciprocal lattice corresponding to $\mathcal{L}$ is defined by

$$
\mathcal{R}=\left\{m \mathbf{u}_{1}+n \mathbf{u}_{2}, m, n \in \mathbb{Z}\right\}
$$

where the basis vectors $\mathbf{u}_{1}, \mathbf{u}_{2} \in \mathbb{R}^{2}$ are defined such that $\mathbf{u}_{i} \cdot \mathbf{e}_{j}=2 \pi \delta_{i j}$, $\forall i, j \in\{1,2\}\left(\delta_{i j}\right.$ is the Kronecker symbol, i.e., $\delta_{i j}=1$ if $i=j$ and $\delta_{i j}=0$ otherwise).

The analysis of waves propagating along the $(x, y)$-plane can be carried out by decomposing the electromagnetic field into two polarisations: Transverse Magnetic $(\mathrm{TM})\left(\mathbf{E}=\left(0,0, E_{z}\right), \mathbf{H}=\left(H_{x}, H_{y}, 0\right)\right)$ and Transverse Electric (TE) $\left(\mathbf{E}=\left(E_{x}, E_{y}, 0\right), \mathbf{H}=\left(0,0, H_{z}\right)\right)$. The field components $v=E_{z}$ and $v=$ $H_{z}$ are chosen as the unknowns for respectively the TM and TE polarisations.

From Maxwell's equations in the frequency domain, the field $v$ must satisfy the Helmholtz equation

$$
\nabla \cdot(p \nabla v(\mathbf{r}))+k^{2} q v(\mathbf{r})=0, \text { in } \mathbb{R}^{2}
$$

where $\mathbf{r}=(x, y)$ denotes the position vector, $k$ is the free space wavenumber, $p=1, q=\varepsilon$ and $v=E_{z}$ in the case of TM polarisation, and $p=1 / \varepsilon, q=1$ and $v=H_{z}$ for TE polarisation.

If $d_{1}$ and $d_{2}$ denote respectively the lengths of the vectors $\mathbf{e}_{1}$ and $\mathbf{e}_{2}$, we assume that the coordinate system is chosen such that

$$
\mathbf{e}_{1}=d_{1}(1,0) \quad \text { and } \quad \mathbf{e}_{2}=d_{2}(\cos \psi, \sin \psi) \text {. }
$$


with $\psi$, the angle between the lattice vectors defined in Fig. 1.

Accordingly, for a square lattice, we have $d_{1}=d_{2}=d$ and $\psi=\pi / 2$, while for a hexagonal lattice $d_{1}=d_{2}=d$ and $\psi=\pi / 3$, where $d$ is the lattice constant, i.e., the smallest distance between the lattice points.

The Bloch mode $[1,18,37,38] v(\mathbf{r})$ associated with the Bloch wave vector $\mathbf{k}_{0} \in$ $\mathbb{R}^{2}$ is a nonzero solution of Eq. (3) which, according to the Bloch Theorem, is the product of the exponential function $e^{i \mathbf{k}_{0} \cdot \mathbf{r}}$ and a function $u(\mathbf{r})$ which is periodic on the lattice $\mathcal{L}$.

In the case of the TM formulation $\left(v=E_{z}\right)$, the substitution $v(\mathbf{r})=e^{i \mathbf{k}_{0} \cdot \mathbf{r}} u(\mathbf{r})$ into (3) gives the following partial differential equation spectral problem:

$$
-\nabla \cdot(\nabla u)-2 i \mathbf{k}_{0} \cdot \nabla u+\left(\mathbf{k}_{0} \cdot \mathbf{k}_{0}\right) u=k^{2} q u .
$$

When, in Eq. (5), we fix the wave vector $\mathbf{k}_{0}$ and solve for the light frequency, we are led to a linear Hermitian eigenproblem having $k^{2}$ as the unknown $[2,27]$. However, when the wavenumber $k$ and one of the two components of

$$
\mathbf{k}_{0}=\left(\alpha_{0}, \beta_{0}\right) \text {, }
$$

$\alpha_{0}$ for instance, are fixed, the resulting eigenproblem is nonlinear since it involves both $\beta_{0}$ and $\beta_{0}^{2}$. Corresponding observations also apply to the TE problem with $v=H_{z}$.

Instead, we consider an alternative method, fixing $k$ and $\alpha_{0}$, and derive a transfer matrix formulation which results in a simple, algebraic eigenproblem for the eigenfunction $v(\mathbf{r})$ which satisfies the Bloch condition

$$
v\left(\mathbf{r}+\mathbf{r}_{m n}\right)=v(\mathbf{r}) e^{i \mathbf{k}_{0} \cdot \mathbf{r}_{m n}}, \forall \mathbf{r} \in \mathbb{R}^{2}
$$

where $\mathbf{r}_{m n}=m \mathbf{e}_{1}+n \mathbf{e}_{2}$, for $m, n \in \mathbb{Z}$, is a general lattice vector.

Let $\Omega$ denote, for example, the following layer of the two dimensional photonic crystal:

$$
\Omega=\left\{t_{1} \mathbf{e}_{1}+t_{2} \mathbf{e}_{2} \mid t_{1} \in \mathbb{R}, t_{2} \in\left[-\frac{1}{2}, \frac{1}{2}\right]\right\}
$$

and, let $\Pi$ and $\Pi^{\prime}$ represent respectively the top and bottom interfaces of $\Omega$ (see Fig. 1), i.e.,

$$
\Pi=\left\{t_{1} \mathbf{e}_{1}+\frac{1}{2} \mathbf{e}_{2} \mid t_{1} \in \mathbb{R}\right\} \text { and } \Pi^{\prime}=\left\{t_{1} \mathbf{e}_{1}-\frac{1}{2} \mathbf{e}_{2} \mid t_{1} \in \mathbb{R}\right\} .
$$


(a)

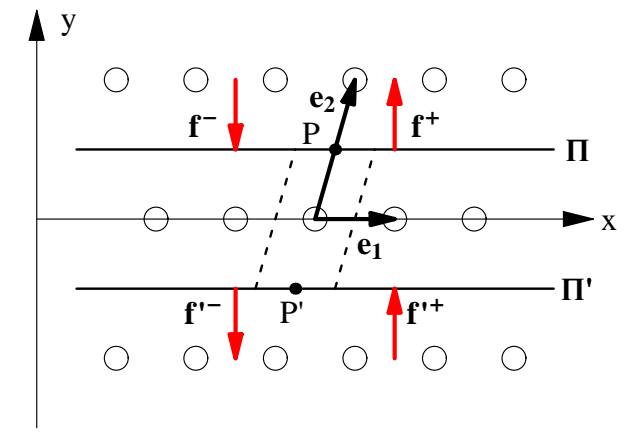

(b)

\begin{tabular}{cccccccccccc}
0 & 0 & 0 & 0 & 0 & 0 & 0 & 0 & 0 & 0 & 0 & 0 \\
0 & 0 & 0 & 0 & 0 & 0 & 0 & 0 & 0 & 0 & 0 & 0 \\
\hline 0 & 0 & 0 & 0 & 0 & 0 & 0 & 0 & 0 & 0 & 0 & 0 \\
\hline 0 & 0 & 0 & 0 & 0 & 0 & 0 & 0 & 0 & 0 & 0 & 0
\end{tabular}

Fig. 1. (a) Geometry of the photonic crystal unit cell, defined by the lattice basis vectors $\mathbf{e}_{1}$ and $\mathbf{e}_{2}$. The phase origins $P$ and $P^{\prime}$ of the fields respectively above $\left(\mathbf{f}^{-}\right.$, $\mathbf{f}^{+}$) and below $\left(\mathbf{f}^{\prime-}, \mathbf{f}^{\prime+}\right)$ the grating are shown. (b) The geometry of a supercell model of a photonic crystal with line defects. The horizontal lines bound a single grating layer of the bulk crystal.

The symbols $\Omega_{0}$ and $\Omega_{0}^{\prime}$ will represent respectively the semi-infinite spaces above and below $\Omega$. From Eqs. (4) and (7), $v(x, y)$ is quasiperiodic with respect to the variable $x$ :

$$
v\left(x+d_{1}, y\right)=v(x, y) e^{i \alpha_{0} d_{1}}, \forall x, y \in \mathbb{R}
$$

We now conceptualise the photonic crystal layer $\Omega$ as a diffraction grating surrounded by a homogeneous medium. The quasiperiodicity imposed by the grating and the incident field leads us to the basis of plane waves

$$
\left\{e^{i\left(\alpha_{m} x \pm \chi_{m} y\right)}\right\}
$$

where $\forall m \in \mathbb{Z}$

$$
\begin{aligned}
& \alpha_{m}=\alpha_{0}+\frac{2 \pi m}{d_{1}}, \\
& \chi_{m}=\left\{\begin{array}{l}
\sqrt{\left(n_{0} k\right)^{2}-\alpha_{m}^{2}}, \text { if }\left(n_{0} k\right)^{2}-\alpha_{m}^{2} \geq 0, \\
i \sqrt{\alpha_{m}^{2}-\left(n_{0} k\right)^{2}}, \text { if }\left(n_{0} k\right)^{2}-\alpha_{m}^{2}<0,
\end{array}\right.
\end{aligned}
$$


with $n_{0}$ denoting the refractive index of the homogeneous medium surrounding $\Omega$. We assume that the homogeneous medium is a lossless dielectric material so that $n_{0}$ is a real and positive number. The fields at the upper interface $\Pi$ and lower interface $\Pi^{\prime}$ of $\Omega$ may be represented respectively by plane wave expansions $\hat{v}$ and $\hat{v}^{\prime}$ as follows [35]:

$$
\begin{aligned}
& \hat{v}=\sum_{m=-\infty}^{+\infty} \chi_{m}^{-\frac{1}{2}}\left[f_{m}^{-} e^{-i \chi_{m}\left(y-y_{0}\right)}+f_{m}^{+} e^{i \chi_{m}\left(y-y_{0}\right)}\right] e^{i \alpha_{m}\left(x-x_{0}\right)} \\
& \hat{v}^{\prime}=\sum_{m=-\infty}^{+\infty} \chi_{m}^{-\frac{1}{2}}\left[f_{m}^{\prime-} e^{-i \chi_{m}\left(y-y_{0}^{\prime}\right)}+f_{m}^{\prime+} e^{i \chi_{m}\left(y-y_{0}^{\prime}\right)}\right] e^{i \alpha_{m}\left(x-x_{0}^{\prime}\right)}
\end{aligned}
$$

where $\left(x_{0}, y_{0}\right) \in \Pi$ and $\left(x_{0}^{\prime}, y_{0}^{\prime}\right) \in \Pi^{\prime}$ are the chosen phase origins, i.e., the points $P$ and $P^{\prime}$ of Fig. 1. For example, when the photonic crystal comprises periodic cylindrical inclusions in an otherwise uniform background medium, we may take $n_{0}$ as the refractive index of the background medium. We also assume that the wavelength does not coincide with a Rayleigh anomaly (also commonly referred as Wood anomaly in the diffraction grating literature) and which occurs at a wavelength for which $\chi_{m} \neq 0$ for some integer $m$, corresponding to the transition of order $m$ from a propagating wave to one that is evanescent.

In Eq. (13), the factor $\chi_{m}^{-1 / 2}$ is chosen to normalise the plane wave amplitudes $f_{m}^{ \pm}$and $f_{m}^{\prime \pm}$ so that energy fluxes may be computed from the square magnitude of the relevant complex amplitudes $[8,9]$. Note that although the variable $y$ in the plane wave expansion (13) does not play any role in the representation of the field $v$ at the interfaces $\Pi$ and $\Pi^{\prime}$ of $\Omega$, the $y$ dependence will be required to enforce the continuity of the tangential field components through derivative conditions across these interfaces.

Although our approach is carried out within the framework of diffraction theory, it is important to point out that in the actual photonic crystal the plane wave expansion (13) is, by construction, valid at the interfaces $\Pi$ and $\Pi^{\prime}$ but its validity beyond these interfaces requires a uniform layer and this requirement had lead to serious limitations on the multipole method. However, since our method needs only the expansion at the interfaces, it can be applied to a general and arbitrary photonic crystal geometry.

If we denote by $\mathbf{f}^{-}, \mathbf{f}^{+}, \mathbf{f}^{\prime-}$ and $\mathbf{f}^{\prime+}$ column vectors whose elements are the plane wave expansion coefficients $f_{m}^{-}, f_{m}^{+}, f_{m}^{\prime-}$ and $f_{m}^{\prime+}$ respectively, and if we assume that the phase origins $\left(x_{0}, y_{0}\right) \in \Pi$ and $\left(x_{0}^{\prime}, y_{0}^{\prime}\right) \in \Pi^{\prime}$ are such that

$$
\left(x_{0}^{\prime}, y_{0}^{\prime}\right)=\left(x_{0}, y_{0}\right)-\mathbf{e}_{2}
$$


then the Bloch condition (7) gives

$$
\mathbf{f}^{\prime-}=\mu \mathbf{f}^{-}, \quad \mathbf{f}^{\prime+}=\mu \mathbf{f}^{+}
$$

where the phase factor $\mu$ is given by

$$
\mu=e^{-i \mathbf{k}_{0} \cdot \mathbf{e}_{2}} .
$$

The set of eigenvalues and eigenvectors of Eq. (15) can be partitioned into two blocks, respectively the downward and upward propagating modes [7]. The propagating modes have eigenvalues of unit magnitude $|\mu|=1$ and the partitioning is done according to the direction of field energy flow, while the evanescent modes are characterised by $|\mu| \neq 1$ and are classified according to the direction of decay of the field, i.e., the downward and upward directions are associated respectively with $|\mu|<1$ and $|\mu|>1$.

The transfer matrix $\mathcal{T}$ relates the fields above and below the grating as follows

$$
\left[\begin{array}{l}
\mathbf{f}^{\prime-} \\
\mathbf{f}^{\prime+}
\end{array}\right]=\mathcal{T}\left[\begin{array}{l}
\mathbf{f}^{-} \\
\mathbf{f}^{+}
\end{array}\right] .
$$

Thus the Bloch factors $\mu=e^{-i \mathbf{k}_{0} \cdot \mathbf{e}_{2}}$ can be obtained as eigenvalues of the transfer matrix $\mathcal{T}$.

In order to benefit from the numerous techniques available in the literature on scattering by diffraction gratings [35], we need to formulate the eigenvalue problem using grating scattering matrices $[7,23,39]$. Thus we introduce the plane wave reflection and transmission scattering matrices of the grating as $\mathbf{R}, \mathbf{T}$ and $\mathbf{R}^{\prime}, \mathbf{T}^{\prime}$, with the two pairs corresponding to incidence from above and below, respectively. For example, $R_{m n}$ denotes the reflected amplitude in the plane wave order $m$ due to the incidence on the top interface of the grating by a unit amplitude plane wave in order $n$ (associated with a direction angle $\theta_{n}$ derived from the grating equation $\left.\sin \theta_{n}=\alpha_{n} /\left(n_{0} k\right)\right)$. Note that these scattering matrices, the form of which are derived in Sec. 3 are of infinite dimension and must be truncated in order to apply the numerical procedures.

The grating scattering matrices can be computed using a variety of numerical techniques for diffraction grating problems such as integral and differential methods [35], and multipole methods $[6,8,9]$ which are appropriate to cylinder gratings. Here, however, we use the finite element method $[13,14,17]$ to derive an efficient and accurate tool that provides the flexibility needed to model arbitrary geometries. As we will see, although the calculation of these matrices involves the solution of multiple grating scattering problems (over 
the set of incident plane wave orders), we may arrange the FEM algorithm in such a way that the most computationally expensive step, associated with the factorisation of FEM matrices, never needs to be repeated.

The grating scattering matrices relate the incoming fields $\mathbf{f}^{-}, \mathbf{f}^{\prime+}$ to the outgoing fields $\mathbf{f}^{+}, \mathbf{f}^{\prime-}$ as follows

$$
\left[\begin{array}{l}
\mathbf{f}^{\prime-} \\
\mathbf{f}^{+}
\end{array}\right]=\left[\begin{array}{cc}
\mathbf{T} & \mathbf{R}^{\prime} \\
\mathbf{R} & \mathbf{T}^{\prime}
\end{array}\right]\left[\begin{array}{l}
\mathbf{f}^{-} \\
\mathbf{f}^{\prime+}
\end{array}\right]
$$

Rearranging the equation defined in Eq. (18) we have

$$
\left[\begin{array}{cc}
\mathbf{I} & -\mathbf{R}^{\prime} \\
\mathbf{0} & \mathbf{T}^{\prime}
\end{array}\right]\left[\begin{array}{l}
\mathbf{f}^{\prime-} \\
\mathbf{f}^{\prime+}
\end{array}\right]=\left[\begin{array}{cc}
\mathbf{T} & \mathbf{0} \\
-\mathbf{R} & \mathbf{I}
\end{array}\right]\left[\begin{array}{l}
\mathbf{f}^{-} \\
\mathbf{f}^{+}
\end{array}\right]
$$

where $\mathbf{I}$ is the identity matrix. Thus the transfer matrix $\mathcal{T}$ is given by

$$
\mathcal{T}=\left[\begin{array}{cc}
\mathbf{I} & -\mathbf{R}^{\prime} \\
\mathbf{0} & \mathbf{T}^{\prime}
\end{array}\right]^{-1}\left[\begin{array}{cc}
\mathbf{T} & \mathbf{0} \\
-\mathbf{R} & \mathbf{I}
\end{array}\right]=\left[\begin{array}{cc}
\mathbf{T}-\mathbf{R}^{\prime} \mathbf{T}^{\prime-1} \mathbf{R} & \mathbf{R}^{\prime} \mathbf{T}^{\prime-1} \\
-\mathbf{T}^{\prime-1} \mathbf{R} & \mathbf{T}^{\prime-1}
\end{array}\right]
$$

The matrices $\mathbf{T}$ and $\mathbf{T}^{\prime}$ are typically ill-conditioned because of the exponential increase or decrease of the evanescent wave components, and so the inversion of the transmission matrices $\mathbf{T}^{\prime}$ in Eq. (20) is quite problematic. It has therefore been necessary to reformulate the eigenproblem in a way that circumvents these instabilities. To do so, we use Eq. (20) to recast the eigenproblem $\mathcal{T} \mathbf{f}=$ $\mu \mathbf{f}$ as

$$
\left[\begin{array}{cc}
\mathbf{T} & \mathbf{0} \\
-\mathbf{R} & \mathbf{I}
\end{array}\right]\left[\begin{array}{l}
\mathbf{f}^{-} \\
\mathbf{f}^{+}
\end{array}\right]=\mu\left[\begin{array}{cc}
\mathbf{I} & -\mathbf{R}^{\prime} \\
\mathbf{0} & \mathbf{T}^{\prime}
\end{array}\right]\left[\begin{array}{l}
\mathbf{f}^{-} \\
\mathbf{f}^{+}
\end{array}\right]
$$

and then apply a shift-and-invert technique to derive the standard linear eigenvalue problem

$$
\left[\begin{array}{cc}
\mathbf{T}-s \mathbf{I} & s \mathbf{R}^{\prime} \\
-\mathbf{R} & \mathbf{I}-s \mathbf{T}^{\prime}
\end{array}\right]^{-1}\left[\begin{array}{cc}
\mathbf{I}-\mathbf{R}^{\prime} \\
\mathbf{0} & \mathbf{T}^{\prime}
\end{array}\right]\left[\begin{array}{l}
\mathbf{f}^{-} \\
\mathbf{f}^{+}
\end{array}\right]=\frac{1}{\mu-s}\left[\begin{array}{l}
\mathbf{f}^{-} \\
\mathbf{f}^{+}
\end{array}\right] .
$$

In our numerical simulations, for example, we set the value of the shift to $s=1$ or $s=2$ and solve the equation (22) using standard numerical codes such as 
the LAPACK library [30] or Mathematica [43]. In passing, we observe that the numerical stability of the generalised eigenvalue problem (21) has also been considered by various authors [11,31]; other numerically stable approaches have also been published, see for instance, [25].

The diagonalised form of the transfer matrix then follows from the eigensystem:

$$
\mathcal{T}=\mathcal{F} \mathcal{L} \mathcal{F}^{-1}, \quad \text { where } \mathcal{F}=\left[\begin{array}{cc}
\mathbf{F}_{-} & \widetilde{\mathbf{F}}_{-} \\
\mathbf{F}_{+} & \widetilde{\mathbf{F}}_{+}
\end{array}\right] \text {and } \mathcal{L}=\left[\begin{array}{cc}
\boldsymbol{\Lambda} & 0 \\
\mathbf{0} & \tilde{\Lambda}
\end{array}\right]
$$

In Eq. (23), the columns of the matrix $\mathcal{F}$ comprise the eigenvectors which constitute the Bloch modes. Its left partition contains the downward propagating modes, with the columns of the constituent matrices $\mathbf{F}_{-}$and $\mathbf{F}_{+}$respectively contain the downward and upward plane wave components $\mathbf{f}_{-}$and $\mathbf{f}_{+}$of the modes of Eq. (15). Correspondingly, the right partition (with quantities distinguished by a tilde symbol ${ }^{\sim}$ ) contains the upward propagating modes. In turn, the diagonal matrix $\mathcal{L}$ comprises the eigenvalues $\mu$, partitioned into downward $(\boldsymbol{\Lambda})$ and upward propagating $(\widetilde{\boldsymbol{\Lambda}})$ modes. As is detailed in Ref. [10], we choose to normalise the Bloch mode matrix $\mathcal{F}$ so that propagating modes carry unit energy, thus reducing the subsequent calculation of energy fluxes to the computation of the square magnitude of the relevant coefficient in the Bloch mode expansion.

To conclude this section, we focus briefly on some properties of transfer matrices and the concomitant, interesting properties that manifest themselves in the distribution of the eigenvalues $\mu$ which are taken up in Sec. 5.2. Before doing so, however, it is important to differentiate between the transfer matrix method which is adopted here, and the more familiar treatment adopted by plane wave expansion methods that solve an operator eigenvalue problem [26] of the form

$$
\Theta \boldsymbol{H}(\boldsymbol{r})=\nabla \times\left(\frac{1}{\varepsilon(\boldsymbol{r})} \nabla \times \boldsymbol{H}(\boldsymbol{r})\right)=k^{2} \boldsymbol{H}(\boldsymbol{r}) .
$$

In computing the Bloch modes from Eq. (24), the eigenvalues $\left(k^{2}\right)$ of which determine the permissible frequencies, we select a Bloch vector $\boldsymbol{k}_{0}$ and then determine a basis of modes (of different frequencies), the orthogonality of which follows from the Hermitian nature of the operator $\Theta$. In contrast, in the treatment of this paper, we select a frequency (or wavelength) and a component of the Bloch vector and proceed to solve the eigenvalue problem

$$
\mathcal{T} f=\mu f
$$


to determine the remaining Bloch factor(s) (via $\mu$ ) and an eigenvector $\boldsymbol{f}$ which characterises the mode. This set of modes is complete and forms a basis in which we can expand electromagnetic fields for the particular frequency. However, the orthogonality properties of the modes are completely different to those derived from the conventional operator eigenvalue treatment [26] and must be deduced from the properties of the transfer matrix $\mathcal{T}$.

There are two key results. The first of these is that, for $\alpha_{0}=0$, the transfer matrix is symplectic [10], i.e.,

$$
\mathcal{T}^{T} \mathcal{Q}_{\mathrm{pw}} \mathcal{T}=\mathcal{Q}_{\mathrm{pw}} \quad \text { where } \quad \mathcal{Q}_{\mathrm{pw}}=\left(\begin{array}{cc}
\boldsymbol{0} & \boldsymbol{Q} \\
-\boldsymbol{Q} & 0
\end{array}\right)
$$

where $\boldsymbol{Q}$ is the reversing permutation, derived by inverting the rows of the identity matrix. The derivation of Eq. (26) for an arbitrary non-symmetric crystal follows from the reciprocity theorem, with the origin of the skewHermitian form of $\mathcal{Q}_{\mathrm{pw}}$ lying in the curl operators of Maxwell's equations. Since its derivation is entirely geometrical in nature, relying only on the reciprocity theorem, it holds for arbitrary materials and thus is applicable to both dielectric and metallic (lossy) structures. It may further be shown that the modes, represented by the columns of the matrix $\mathcal{F}(23)$, satisfy

$$
\mathcal{F}^{H} \mathcal{Q}_{\mathrm{pw}} \mathcal{F}=\left(\begin{array}{cc}
\mathbf{0} & \boldsymbol{I} \\
-\boldsymbol{I} & 0
\end{array}\right)
$$

when appropriately normalised.

The second key relation constitutes a generalisation [10] of unitarity, with the generalisation taking into account the need for evanescent plane waves in addition to propagating plane waves. That is,

$$
\mathcal{T}^{H} \mathcal{I}_{\mathrm{pw}} \mathcal{T}=\mathcal{I}_{\mathrm{pw}} \quad \text { where } \quad \mathcal{I}_{\mathrm{pw}}=\left(\begin{array}{cc}
\mathbf{I}_{p} & -i \mathbf{I}_{\bar{p}} \\
i \mathbf{I}_{\bar{p}} & -\mathbf{I}_{p}
\end{array}\right)
$$

in which $\mathbf{I}_{p}$ denotes a diagonal matrix whose rows and columns designate the plane wave orders and whose diagonal elements are 1 for propagating plane waves and 0 otherwise. The matrix $\mathbf{I}_{\bar{p}}=\mathbf{I}-\mathbf{I}_{p}$ is its complement, containing unit diagonal elements only for the evanescent plane waves. From this, one 
may derive a modal orthogonality relation

$$
\mathcal{F}^{H} \mathcal{I}_{\mathrm{pw}} \mathcal{F}=\mathcal{I}_{\mathrm{bm}} \quad \text { where } \quad\left(\begin{array}{cc}
\mathbf{I}_{m} & -i \mathbf{I}_{\bar{m}} \\
i \mathbf{I}_{\bar{m}} & -\mathbf{I}_{m}
\end{array}\right)
$$

in which $\mathbf{I}_{m}$ and $\mathbf{I}_{\bar{m}}$ denote the Bloch mode analogues of the corresponding plane wave forms $\mathbf{I}_{p}$ and $\mathbf{I}_{\bar{p}}$, and have unit diagonal entries denoting the place of propagating and evanescent Bloch modes. The derivation of Eq. (28) follows from an energy argument and thus Eqs (28) and (29) hold only for lossless systems but are valid for arbitrary values of $\alpha_{0} \in \mathbb{R}$.

\section{Variational formulation of the grating scattering problem}

\subsection{Overview and nomenclature}

Although in the case of a photonic crystal layer $\Omega$ we take the semi-infinite homogeneous media above and below to be identical, we will derive the finite element scattering matrix algorithm for the general case in which the refractive indices of the media above and below differ (with a superscripted prime (') referring to quantities associated with the homogeneous medium below the grating). Also, to simplify the presentation of the FEM, we will solve the grating scattering problem using unscaled plane wave coefficients, i.e., without the scaling factors $\chi_{m}^{-1 / 2}$ that appears in Eq. (13), and then rescale the coefficients to introduce the energy normalisation at the conclusion of the derivation.

For a given value of $\alpha_{0} \in \mathbb{R}$ and for an arbitrary order $m \in \mathbb{Z}$, the plane wave parameters $\alpha_{m}, \chi_{m}$ and $\chi_{m}^{\prime}$ are defined by Eq. (11); for $\chi_{m}^{\prime}$, the refractive index value $n_{0}$ is replaced by $n_{0}^{\prime}$ in Eq. (11). The reflection and transmission scattering matrices that characterise the diffraction properties of the grating, will be filled column-wise, with each column corresponding to a particular incidence order. The scattering matrices can be determined by computing, for each incident order $n$ (including evanescent orders) from both above $\left(\exp \left[i \alpha_{n}\left(x-x_{0}\right)-i \chi_{n}\left(y-y_{0}\right)\right]\right)$ and below $\left(\exp \left[i \alpha_{n}\left(x-x_{0}^{\prime}\right)+i \chi_{n}^{\prime}\left(y-y_{0}^{\prime}\right)\right]\right)$, the reflected and transmitted amplitudes of the outgoing plane wave fields.

For example, if the downward propagating plane wave

$$
v_{n}^{\mathrm{inc}}=e^{i \alpha_{n}\left(x-x_{0}\right)-i \chi_{n}\left(y-y_{0}\right)}
$$

is incident from above the grating, the total field propagating over the semiinfinite domains $\Omega_{0}$ and $\Omega_{0}^{\prime}$ can be represented respectively by the following 
plane wave expansions

$$
\left\{\begin{array}{l}
v_{n}^{+}(x, y)=\sum_{m=-\infty}^{+\infty}\left[\delta_{m n} e^{-i \chi_{m}\left(y-y_{0}\right)}+r_{m n} e^{i \chi_{m}\left(y-y_{0}\right)}\right] e^{i \alpha_{m}\left(x-x_{0}\right)} \\
v_{n}^{-}(x, y)=\sum_{m=-\infty}^{+\infty} t_{m n} e^{i\left(\alpha_{m}\left(x-x_{0}^{\prime}\right)-\chi_{m}^{\prime}\left(y-y_{0}^{\prime}\right)\right)}
\end{array}\right.
$$

where $r_{m n}$ and $t_{m n}$ are respectively the coefficients of the outgoing reflected and transmitted plane wave coefficients, and $\delta_{m n}$ denotes the Kronecker symbol.

Similarly, for incidence from below the grating, we have

$$
v_{n}^{\text {inc }}=e^{i \alpha_{n}\left(x-x_{0}^{\prime}\right)+i \chi_{n}^{\prime}\left(y-y_{0}^{\prime}\right)}
$$

and also the plane wave expansions denoting the field above and below:

$$
\left\{\begin{array}{l}
v_{n}^{\prime+}(x, y)=\sum_{m=-\infty}^{+\infty} t_{m n}^{\prime} e^{i\left(\alpha_{m}\left(x-x_{0}\right)+\chi_{m}\left(y-y_{0}\right)\right)} \\
v_{n}^{\prime-}(x, y)=\sum_{m=-\infty}^{+\infty}\left[\delta_{m n} e^{i \chi_{m}^{\prime}\left(y-y_{0}^{\prime}\right)}+r_{m n}^{\prime} e^{-i \chi_{m}^{\prime}\left(y-y_{0}^{\prime}\right)}\right] e^{i \alpha_{m}\left(x-x_{0}^{\prime}\right)}
\end{array}\right.
$$

\subsection{Plane wave incidence from above the grating: variational formulation}

From the periodicity of the structure and from the quasi-periodicity of the incident plane wave, the solution $v_{n}$ has to be quasi-periodic :

$$
v_{n}\left(x+d_{1}, y\right)=e^{i \alpha_{0} d_{1}} v_{n}(x, y), \quad \forall(x, y) \in \Omega .
$$

Inside the grating area $\Omega$, the field must satisfy the Helmholtz equation

$$
\nabla \cdot\left(p \nabla v_{n}\right)+k^{2} q v_{n}=0 .
$$

The continuity of the tangential components of the electric and magnetic fields across the interfaces $\Pi$ and $\Pi^{\prime}$ implies that $v_{n}$ and $p\left(\partial v_{n} / \partial \nu\right)$ must be continuous. Accordingly, from Eq. (31), the field and derivative boundary conditions are:

$$
\left\{\begin{array}{l}
v_{n}\left(x, y_{0}\right)=\psi_{n}(x)+\sum_{m=-\infty}^{+\infty} r_{m n} \psi_{m}(x), \text { on } \Pi \\
v_{n}\left(x, y_{0}^{\prime}\right)=\sum_{m=-\infty}^{+\infty} t_{m n} \psi_{m}^{(\prime)}(x), \text { on } \Pi^{\prime}
\end{array}\right.
$$


and

$$
\left\{\begin{array}{l}
p \frac{\partial v_{n}\left(x, y_{0}\right)}{\partial \nu}=-i p_{0} \chi_{n} \psi_{n}(x)+i p_{0} \sum_{m=-\infty}^{+\infty} \chi_{m} r_{m n} \psi_{m}(x), \text { on } \Pi, \\
p \frac{\partial v_{n}\left(x, y_{0}^{\prime}\right)}{\partial \nu}=i p_{0}^{\prime} \sum_{m=-\infty}^{+\infty} \chi_{m}^{\prime} t_{m n} \psi_{m}^{(\prime)}(x), \text { on } \Pi^{\prime},
\end{array}\right.
$$

where

$$
\psi_{m}(x)=e^{i \alpha_{m}\left(x-x_{0}\right)} \quad \text { and } \quad \psi_{m}^{(\prime)}(x)=e^{i \alpha_{m}\left(x-x_{0}^{\prime}\right)}, \forall x \in \mathbb{R}
$$

and where $p_{0}$ and $p_{0}^{\prime}$ are the constant values of the function $p$ over $\Omega_{0}$ and $\Omega_{0}^{\prime}$ respectively, with the operator $\partial / \partial \nu$ denoting the outward normal derivative.

To obtain the variational formulation of the problem (35)-(37), we must first introduce the Sobolev space of quasi-periodic functions $H_{\left(\alpha_{0}\right)}^{1}(\Omega)$, defined as the closure of the set of smooth functions

$$
C_{\left(\alpha_{0}\right)}^{\infty}(\bar{\Omega})=\left\{v \in C^{\infty}(\bar{\Omega}) \mid v\left(x+d_{1}, y\right)=e^{i \alpha_{0} d_{1}} v(x, y), \forall(x, y) \in \Omega\right\}
$$

with respect to the norm $\|\cdot\|_{1, Q}$ of the Sobolev space $H^{1}(Q)$ where $Q$ is a unit cell of the $x$-periodic domain $\Omega$. For example, $Q$ can be chosen as in Fig. 1. For the remainder of the paper, we shall regard the domain $\Omega$ as the unit cell $Q$ with quasi-periodic boundary conditions.

The variational formulation is obtained in two steps. First we multiply Eq. (35) by the conjugate of test functions $w \in H_{\left(\alpha_{0}\right)}^{1}(\Omega)$ and integrate by parts, taking into account the boundary conditions (37) and the quasi-periodicity. Then we multiply Eq. (36) by plane wave exponential functions $\psi_{m}^{*}(x)$ or $\psi_{m}^{(\prime)}{ }^{*}(x)$, and apply their orthogonality. This leads to the following weak formulation : 
Given $n \in \mathbb{Z}$, find the field $v_{n} \in H_{\left(\alpha_{0}\right)}^{1}(\Omega)$, and plane wave coefficients $r_{m n}$ and $t_{m n}$, for $m \in \mathbb{Z}$, such that

$$
\left\{\begin{array}{l}
\int_{\Omega}\left(-p\left(\nabla v_{n}\right) \cdot\left(\nabla w^{*}\right)+k^{2} q v_{n} w^{*}\right) d x d y \\
\quad+i p_{0} \sum_{m=-\infty}^{+\infty} \chi_{m} r_{m n} \int_{x_{0}-d_{1} / 2}^{x_{0}+d_{1} / 2} \psi_{m}(x) w^{*}\left(x, y_{0}\right) d x \\
\quad-i p_{0}^{\prime} \sum_{m=-\infty}^{+\infty} \chi_{m}^{\prime} t_{m n} \int_{x_{0}^{\prime}+d_{1} / 2}^{\prime} \psi_{m}^{(\prime)}(x) w^{*}\left(x, y_{0}^{\prime}\right) d x \\
=i p_{0} \chi_{n} \int_{x_{1} / 2}^{x_{0}+d_{1} / 2} \psi_{n}(x) w^{*}\left(x, y_{0}\right) d x, \quad \forall w \in H_{\left(\alpha_{0}\right)}^{1}(\Omega) \\
d_{1} r_{m n}-\int_{x_{0}-d_{1} / 2}^{x_{0}-d_{1} / 2} v_{n}\left(x, y_{0}\right) \psi_{m}^{*}(x) d x=-d_{1} \delta_{m, n}, \quad \forall m \in \mathbb{Z}, \\
d_{1} t_{m n}-\int_{x_{0}^{\prime}-d_{1} / 2}^{x_{1} / 2} v_{n}\left(x, y_{0}^{\prime}\right) \psi_{m}^{(\prime)}(x) d x=0, \quad \forall m \in \mathbb{Z},
\end{array}\right.
$$

where ${ }^{*}$ denotes the complex-conjugate operator.

The theoretical analysis of variational problem (40) has been the subject of many papers, e.g., Refs. [3,19]. For all but possibly a discrete set of frequencies, the existence and uniqueness of a solution of Eq. (40) has been proved and the convergence of the finite element solution has been established [3,19]. In most of the analytical studies, which use a nonlocal boundary operator (Dirichletto-Neumann map), Eq. (40) is further transformed into a more compact form where the theory of elliptic boundary value problems may be applied. However in order to avoid a significant loss of sparsity in the FEM matrices, it is appropriate to discretise directly Eq. (40).

We also consider the case of incidence from below, with the details differing from $\mathrm{Eq}(40)$ only by the fact the incident source terms and the reflection matrix coefficients $r_{m n}^{\prime}$ are attached the lower interface while the transmission matrix coefficients $t_{m n}^{\prime}$ appear on the upper interface.

\section{Finite element computation of the grating scattering matrices}

We use a standard quadratic finite element method to construct an approximating finite dimensional subspace $V_{h}$ of the space $H_{\left(\alpha_{0}\right)}^{1}(\Omega)$. More details 
concerning the finite element procedures can be found in Ref. [12]. The parameter $h$ refers to the maximum diameter of the triangles in the finite element mesh associated with $V_{h}$. The grating unit cell is represented by an $x$-periodic triangular FEM mesh. Here $N_{h}$ refers to the dimension of the FEM space $V_{h}$ and $\left\{\phi_{m}\right\}_{m=1}^{N_{h}}$ is the nodal basis of the subspace $V_{h}$. The quasi-periodicity condition must be enforced on the basis functions associated with nodes lying on the left and the right periodic boundaries of the FEM mesh; the other functions are zero on these boundaries thus are not affected by the quasi-periodic boundary condition. The approximate solution $v_{n h}$ is expanded in terms of the basis functions as

$$
v_{n h}=\sum_{m=1}^{N_{h}} v_{n m} \phi_{m} .
$$

To obtain a numerical solution, we truncate the plane wave expansion to a finite number of plane wave orders $m \in[-N, N]$, where the integer truncation parameter $N$ is chosen to be sufficient to ensure the accuracy of the computed solution. $N$ is usually chosen such that the truncated Rayleigh expansion includes all the propagating orders and a number of evanescent diffraction orders, sufficient to ensure that the high frequency components of the field are suitably accommodated. In our calculations, we include those evanescent waves that do not increase or decrease by more than a factor of $\epsilon>0$ across the grating thickness, i.e.,

$$
\exp \left(-\left|\chi_{n}\right|\left(y_{0}-y_{0}^{\prime}\right)\right)>\epsilon \quad \text { or } \quad \exp \left(-\left|\chi_{n}^{\prime}\right|\left(y_{0}-y_{0}^{\prime}\right)\right)>\epsilon \text {. }
$$

For the examples we consider in this paper, we choose $\epsilon=10^{-2}$ and, for the normalised frequency range $0<d / \lambda \lesssim 0.7$ in our examples, we observe no significant improvement of the numerical solution by increasing the number of plane wave orders further.

We first consider the case of plane wave incidence from above and apply the Galerkin procedure to the variational problem by substituting the trial function $v_{n h}$ of Eq. (41) into Eq. (40) to derive the following linear system

$$
\left[\begin{array}{ccc}
\mathbf{M}_{v v} & \mathbf{M}_{v r} & \mathbf{M}_{v t} \\
\mathbf{M}_{r v} & d_{1} \mathbf{I} & \mathbf{0} \\
\mathbf{M}_{t v} & \mathbf{0} & d_{1} \mathbf{I}
\end{array}\right]\left[\begin{array}{l}
\mathbf{v}_{n} \\
\mathbf{r}_{n} \\
\mathbf{t}_{n}
\end{array}\right]=\left[\begin{array}{c}
\mathbf{f}_{v n} \\
\mathbf{f}_{r n} \\
\mathbf{0}
\end{array}\right]
$$

where the unknown vectors $\mathbf{v}_{n}, \mathbf{r}_{n}$ and $\mathbf{t}_{n}$ contain respectively the FEM basis coefficients, the plane wave coefficients $r_{m n}$ and $t_{m n} ; \mathbf{I}$ is the identity matrix of order $(2 N+1), \mathbf{0}$ is the null matrix or the null vector of order $(2 N+1)$ 
and the other submatrices of the system (43) are defined as

$$
\begin{aligned}
& \left(M_{v v}\right)_{m s}=\int_{\Omega}\left(-p\left(\nabla \phi_{s}\right) \cdot\left(\nabla \phi_{m}^{*}\right)+k^{2} q \phi_{s} \phi_{m}^{*}\right) d x d y, \\
& m, s=1, \ldots, N_{h}, \\
& \left(M_{v r}\right)_{m s}=i p_{0} \chi_{s-N-1} \int_{x_{0}-d_{1} / 2}^{x_{0}+d_{1} / 2} \psi_{\alpha_{s-N-1}}(x) \phi_{m}^{*}\left(x, y_{0}\right) d x \\
& m=1, \ldots, N_{h}, s=1, \ldots,(2 N+1), \\
& x_{0}^{\prime}+d_{1} / 2 \\
& \left(M_{v t}\right)_{m s}=-i p_{0}^{\prime} \chi_{s-N-1}^{\prime} \int_{x_{0}^{\prime}-d_{1} / 2} \psi_{\alpha_{s-N-1}}^{(\prime)}(x) \phi_{m}^{*}\left(x, y_{0}^{\prime}\right) d x \text {, } \\
& m=1, \ldots, N_{h}, s=1, \ldots,(2 N+1), \\
& \left(M_{r v}\right)_{m s}=-\int_{x_{0}-d_{1} / 2}^{x_{0}+d_{1} / 2} \phi_{s}\left(x, y_{0}\right) \psi_{\alpha_{m-N-1}}^{*}(x) d x \text {, } \\
& m=1, \ldots,(2 N+1), s=1, \ldots, N_{h}, \\
& x_{0}^{\prime}+d_{1} / 2 \\
& \left(M_{t v}\right)_{m s}=-\int_{x_{0}^{\prime}-d_{1} / 2}^{x_{0}+d_{1} / 2} \phi_{s}\left(x, y_{0}^{\prime}\right) \psi_{\alpha_{m-N-1}}^{(\prime) *}(x) d x \\
& m=1, \ldots,(2 N+1), s=1, \ldots, N_{h} ;
\end{aligned}
$$

The coefficients of the source terms are

$$
\begin{aligned}
& \left(f_{v n}\right)_{m}=i p_{0} \chi_{n} \int_{x_{0}-d_{1} / 2}^{x_{0}+d_{1} / 2} \psi_{n}(x) \phi_{m}^{*}\left(x, y_{0}\right) d x, \quad m=1, \ldots, N_{h}, \\
& \left(f_{r n}\right)_{m}=-d_{1} \delta_{(m-N-1),(n-N-1)}, \quad m=1, \ldots,(2 N+1) .
\end{aligned}
$$

For plane wave incidence on the lower grating interface, the right hand side of Eq. (43) becomes $\left[\mathbf{f}^{\prime}{ }_{v n}, \mathbf{0}, \mathbf{f}^{\prime}{ }_{r n}\right]^{T}$ with

$$
\begin{aligned}
& \left(f_{v n}^{\prime}\right)_{m}=i p_{0}^{\prime} \chi_{n} \int_{x_{0}^{\prime}-d_{1} / 2}^{x_{0}^{\prime}+d_{1} / 2} \exp \left(i \alpha_{n}\left(x-x_{0}^{\prime}\right)\right) \phi_{m}^{*}\left(x, y_{0}^{\prime}\right) d x, \quad m=1, \ldots, N_{h}, \\
& \left(f_{r n}^{\prime}\right)_{m}=-d_{1} \delta_{(m-N-1),(n-N-1)}, \quad m=1, \ldots,(2 N+1) .
\end{aligned}
$$




\subsection{Solution of the finite element system}

The submatrix $\mathbf{M}_{v v}$ is a classic finite element matrix and has a sparse band matrix profile. However, since the exponential functions $\psi_{m}(x)$ and $\psi_{m}^{(\prime)}(x)$ vary along the boundaries $\Pi$ and $\Pi^{\prime}$, the columns of $\mathbf{M}_{v r}$ and $\mathbf{M}_{v t}$, and the rows of $\mathbf{M}_{r v}$ and $\mathbf{M}_{t v}$, have a relatively large number of nonzero elements because coefficients for the degrees of freedom corresponding to FEM nodes located on $\Pi$ or $\Pi^{\prime}$, in general, are nonzero. The coefficient matrix of the system (43) is usually referred to as a bordered matrix because of the shape of its nonzero elements; its bandwidth can be quite large. This is an unfavourable situation since most existing finite element tools are optimised for sparse and relatively small bandwidth matrices. Accordingly, it was necessary to derive a method that would allow us to efficiently solve Eq. (43) using algorithms for standard finite element sparse matrices or for low dimension dense matrices.

Expressing $\mathbf{v}_{n}$ in term of $\mathbf{r}_{n}$ and $\mathbf{t}_{n}$, we have

$$
\mathbf{v}_{n}=\mathbf{M}_{v v}^{-1} \mathbf{f}_{v n}-\mathbf{M}_{v v}^{-1} \mathbf{M}_{v r} \mathbf{r}_{n}-\mathbf{M}_{v v}^{-1} \mathbf{M}_{v t} \mathbf{t}_{n}
$$

and substituting this into Eq. (43) leads to the following low dimension and dense matrix system

$$
\left[\begin{array}{ll}
\hat{\mathbf{M}}_{r r} & \hat{\mathbf{M}}_{r t} \\
\hat{\mathbf{M}}_{t r} & \hat{\mathbf{M}}_{t t}
\end{array}\right]\left[\begin{array}{l}
\mathbf{r}_{n} \\
\mathbf{t}_{n}
\end{array}\right]=\left[\begin{array}{l}
\hat{\mathbf{f}}_{r n} \\
\hat{\mathbf{f}}_{t n}
\end{array}\right]
$$

in which the submatrices and the vectors of Eq. (48) are given by

$$
\begin{aligned}
& \hat{\mathbf{M}}_{r r}=d_{1} \mathbf{I}-\mathbf{M}_{r v} \mathbf{M}_{v v}^{-1} \mathbf{M}_{v r}, \\
& \hat{\mathbf{M}}_{r t}=-\mathbf{M}_{r v} \mathbf{M}_{v v}^{-1} \mathbf{M}_{v t}, \\
& \hat{\mathbf{M}}_{t t}=d_{1} \mathbf{I}-\mathbf{M}_{t v} \mathbf{M}_{v v}^{-1} \mathbf{M}_{v t} \\
& \hat{\mathbf{M}}_{t r}=-\mathbf{M}_{t v} \mathbf{M}_{v v}^{-1} \mathbf{M}_{v r}
\end{aligned}
$$

and

$$
\begin{aligned}
& \hat{\mathbf{f}}_{r n}=\mathbf{f}_{r n}-\mathbf{M}_{r v} \mathbf{M}_{v v}^{-1} \mathbf{f}_{v n} \\
& \hat{\mathbf{f}}_{t n}=-\mathbf{M}_{t v} \mathbf{M}_{v v}^{-1} \mathbf{f}_{v n} .
\end{aligned}
$$

Similarly, for the case of incidence from below by an upward propagating wave 
we have

$$
\left[\begin{array}{ll}
\hat{\mathbf{M}}_{r r} & \hat{\mathbf{M}}_{r t} \\
\hat{\mathbf{M}}_{t r} & \hat{\mathbf{M}}_{t t}
\end{array}\right]\left[\begin{array}{l}
\mathbf{t}^{\prime}{ }_{n} \\
\mathbf{r}_{n}^{\prime}
\end{array}\right]=\left[\begin{array}{l}
\hat{\mathbf{f}}_{t n}^{\prime} \\
\hat{\mathbf{f}}_{r n}^{\prime}
\end{array}\right]
$$

where

$$
\begin{aligned}
& \hat{\mathbf{f}}_{t n}^{\prime}=\mathbf{f}_{t n}^{\prime}-\mathbf{M}_{r v} \mathbf{M}_{v v}^{-1} \mathbf{f}_{v n}^{\prime}, \\
& \hat{\mathbf{f}}_{r n}^{\prime}=-\mathbf{M}_{t v} \mathbf{M}_{v v}^{-1} \mathbf{f}_{v n}^{\prime} .
\end{aligned}
$$

In Refs. $[13,17]$ we developed a computationally efficient approach for constructing the solution of Eqs. (48) and (51) in order to determine the unknowns $\mathbf{r}_{n}$ and $\mathbf{t}_{n}$ from which the field $\mathbf{v}_{n}$ can be subsequently reconstructed using Eq. (47). In particular, for Eqs. (47), (49), (50) and (52), the matrix products involving $\mathbf{M}_{v v}^{-1}$ are computed using an LU factorisation of the FEM matrix $\mathbf{M}_{v v}$. During the LU decomposition, coefficients which are zero in the original coefficient matrix of Eq. (43) may become nonzero (fill-in). The amount of fill-in can be reduced by using an appropriate numbering of the FEM mesh nodes.

Finally, we observe that, for a lossless material, the coefficients of the finite element matrix $M_{v v}$, as given by Eq. (44) 1 , are real numbers when the Bloch factor $e^{i \alpha_{0} d_{1}}$ in Eq. (34) is equal to 1 (periodic boundary condition) or -1 (antiperiodic boundary condition) and, accordingly, we can avoid complex number computation by solving separately for the real part and imaginary part. Even for the general case, a coefficient $\left(M_{v v}\right)_{m s}$ can have non zero imaginary part only if either the basis functions $\phi_{s}$ or $\phi_{m}$ is associated with a node on the left or right boundaries because, as we have seen in the first paragraph of this section, only nodal functions based on these boundaries are subjected to the quasi-periodic condition; typically most of the elements of $M_{v v}$ will be real numbers and computation performance can be significant improved by taking advantage of that fact to reduce complex number operations; this can be achieved by reordering the basis functions so that we can assume that the last rows and columns $M_{v v}$ correspond to the complex valued basis functions; we will then have a situation similar to the one in Eq (43): a large central submatrix has a structure (real coefficients) that we wish to use but it is bordered by submatrices which do not enjoy the same structure. To solve this new bordered system, we can easily adapt the approach we develop for the system (43). 


\subsection{Computation of the scattering matrices}

Let $\widetilde{\mathbf{R}}, \widetilde{\mathbf{T}}, \widetilde{\mathbf{R}^{\prime}}$ and $\widetilde{\mathbf{T}}^{\prime}$ denote the matrices whose columns are respectively the vectors $\mathbf{r}_{n}, \mathbf{t}_{n}, \mathbf{r}_{n}^{\prime}, \mathbf{t}_{n}^{\prime}$, for $n=-N, \ldots, N$.

From (48) and (51), the scattering matrices $\widetilde{\mathbf{R}}, \widetilde{\mathbf{T}}, \widetilde{\mathbf{R}^{\prime}}$ and $\widetilde{\mathbf{T}}^{\prime}$ satisfy the following equation

$$
\left[\begin{array}{ll}
\hat{\mathbf{M}}_{r r} & \hat{\mathbf{M}}_{r t} \\
\hat{\mathbf{M}}_{t r} & \hat{\mathbf{M}}_{t t}
\end{array}\right]\left[\begin{array}{cc}
\widetilde{\mathbf{R}} & \widetilde{\mathbf{T}}^{\prime} \\
\widetilde{\mathbf{T}} & \widetilde{\mathbf{R}}^{\prime}
\end{array}\right]=\left[\begin{array}{cc}
\hat{\mathbf{M}}_{r r}-2 d_{1} \mathbf{I} & \hat{\mathbf{M}}_{r t} \\
\hat{\mathbf{M}}_{t r} & \hat{\mathbf{M}}_{t t}-2 d_{1} \mathbf{I}
\end{array}\right]
$$

In particular, the right hand side matrix can be obtained easily from the system matrix and thus computation time can be saved by avoiding the direct calculation of the right hand side vectors. The matrix system (53) can be solved using, for instance, the LAPACK library [30] which can handle systems of linear equations with multiple right hand sides.

Now, if we scale the plane wave coefficients of the incident, reflected and transmitted fields in the same way as in Eq. (13), the corresponding scattering matrices $\mathbf{R}, \mathbf{T}, \mathbf{R}^{\prime}, \mathbf{T}^{\prime}$ are given by the similarity transformation

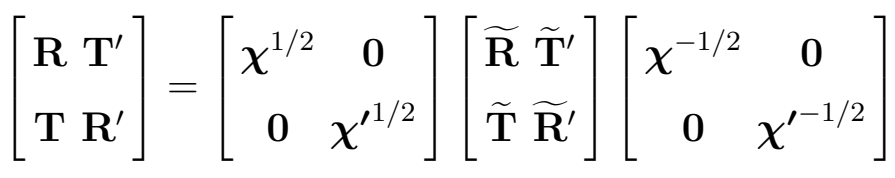

where $\chi$ and $\chi^{\prime}$ are the following diagonal matrices:

$$
\chi=\operatorname{diag}\left[\chi_{m}\right] \text { and } \chi^{\prime}=\operatorname{diag}\left[\chi_{m}^{\prime}\right]
$$

This normalisation is used to simplify the calculation of energy quantities. For example, if $n, m \in \mathbb{Z}$ correspond to propagating diffracted orders, then the fraction of energy (diffraction efficiency) reflected into the $m^{\text {th }}$ order by an incident downward propagating plane wave of order $n$, is given by $\left|\widetilde{\mathbf{R}}_{m n}\right|^{2} \chi_{m} / \chi_{n}$ in term of the unscaled matrix, or simply $\left|\mathbf{R}_{m n}\right|^{2}$ when using the normalised matrix. 
(a)

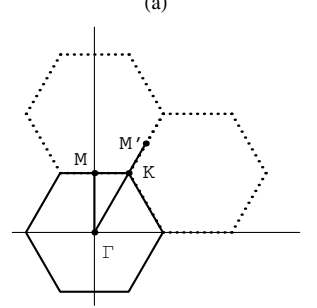

(c)

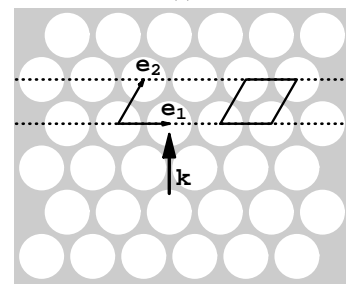

(b)

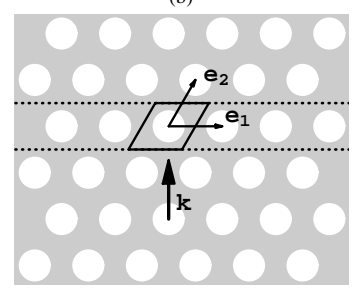

(d)

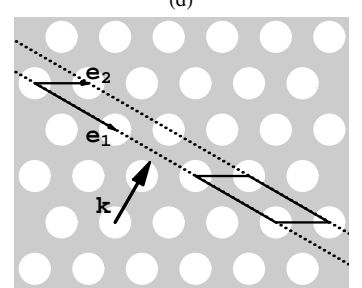

Fig. 2. Hexgonal lattice photonic crystals: (a) The first Brillouin zone and two of its replicates in the reciprocal space. (b) and (c) show a grating layer (area between the dotted lines) and a unit cell (parallelogram) when the wave vector $\mathbf{k}_{0}$ is parallel to the $\Gamma-M$ direction. (d) presents a grating layer and a unit cell when $\mathbf{k}_{0}$ is parallel to $\Gamma-K$. The coordinates of the vectors are given in Table 3 .

\section{$5 \quad$ Numerical examples}

\subsection{Convergence of the method}

We turn now to discuss our investigation of the convergence properties of the numerical method and consider a hexagonal lattice of circular inclusions. The parameters of the problem are: period of the grating $d_{1}=d$ ( $d$ being the lattice constant), background refractive index $n_{b}=3$, cylinder refractive index $n_{c}=1$, radius of the cylinders $a / d=0.3$, free space wavelength $\lambda / d=2$, and transverse Bloch wave vector $\alpha_{0}=\pi /(6 d)$ (see Eq. (6)).

Two possible choices of grating layers, with period $d_{1}=d$, are shown in Fig 2 (b) and (c). We use the layer of Fig 2 (b) for the results presented in this section. We will give more details about Fig. 2 later in section 5.3.

Let $\mathcal{M}_{h}$ be a finite element triangulation of a unit cell, with the parameter $h$ referring to the maximum diameter of the triangles in $\mathcal{M}_{h}$. Let $\mu_{h}$ denote an approximation to the eigenvalue $\mu$ computed using an FEM of order $p$ on the mesh $\mathcal{M}_{h}$. Then, if the eigenfunction associated with the eigenvalue $\mu$ is sufficiently regular and under additional conditions on the quality of the FEM meshes, standard convergence results for elliptic eigenvalue problems [12] predict that $\mu_{h}$ converges to $\mu$ with a convergence rate of $2 p$, i.e., $\left|\mu_{h}-\mu\right| \leq C h^{2 p}$ where the coefficient $C$ does not depend on $h$. 
Table 1

Computational timings for various FEM meshes $\mathcal{M}_{h_{j}}$ : number of triangles $(N T)$, number of nodes $(N N$, vertices and edge midpoints), plane wave truncation parameter $(N)$ and computation time ( $C P U$, for isoparametric FEM).

\begin{tabular}{ccccc}
\hline & $N T$ & $N N$ & $N$ & $C P U$ (sec.) \\
\hline $\mathcal{M}_{h_{0}}$ & 220 & 481 & 6 & 1 \\
$\mathcal{M}_{h_{1}}$ & 966 & 2021 & 11 & 1.5 \\
$\mathcal{M}_{h_{2}}$ & 3722 & 7621 & 20 & 7.75 \\
$\mathcal{M}_{h_{3}}$ & 14792 & 29945 & 20 & 70.2 \\
$\mathcal{M}_{h_{4}}$ & 59948 & 120625 & 20 & 1025 \\
\hline
\end{tabular}

To evaluate the convergence rate of our approximation we use an FEM of order $p=2$ and generate a sequence of 5 successively refined meshes $\mathcal{M}_{h_{0}}, \mathcal{M}_{h_{1}}$, $\mathcal{M}_{h_{2}}, \mathcal{M}_{h_{3}}$ and $\mathcal{M}_{h_{4}}$ with $h_{j}=h_{0} / 2^{j}$, for $j=1,2,3,4$, using the commonly available software "Gmsh" [22]. Some details about the computation with these meshes are given in Table 1 . The initial coarse mesh $\mathcal{M}_{h_{0}}$ is shown in Figure 3 (a).

For both TE and TM polarisations, we computed on each mesh $\mathcal{M}_{h_{j}}$ those eigenvalues $\mu_{h_{j}}^{i}$ such that $1 / 5<\left|\mu_{h_{j}}^{i}\right|<5$. The number of these eigenvalues is 6 for either polarisation. Since the exact solution is not known, we choose to approximate the error by $\left|\mu_{h_{j}}^{i}-\mu^{i}\right| \approx\left|\mu_{h_{j}}^{i}-\mu_{h_{j+1}}^{i}\right|$ for $j=0,1,2$, 3. In Fig. 3 we plot $e(j)=\max _{i}\left\{\left|\mu_{h_{j}}^{i}-\mu_{h_{j+1}}^{i}\right|\right\}$ as a function of $j \in\{0,1,2,3\}$. In Fig. 3 (b) we use standard FEM meshes with rectilinear triangles and discover that the convergence rate is 2 , lower than the theoretical convergence order $2 p=4$. This suboptimal convergence can be attributed directly to the approximation of the circular cylinder interface by a polygon. Indeed, when we use curved triangles (isoparametric FEM triangles) to approximate the curved interface by piecewise quadratic polynomials, we obtain the optimal convergence order $2 p=4$ shown in Fig. 3 (c). Accordingly we will adopt the isoparametric FEM for our remaining numerical examples.

Before concluding our discussion of convergence, we consider briefly the effect of plane wave parameters on our calculations. This is of particular importance in device applications in which the regular photonic crystal lattice is perturbed by the introduction of defects to form waveguides, resonant cavities, couplers and the like. All such applications require the device to be operated in a band gap and, for example, the removal of a entire line of scatterers to form a waveguide introduces a band of defect states that enables the channel to guide the light. This is exemplified in Fig. 4 (b) which displays the dispersion curve for the defect mode corresponding to a waveguide introduced into a square symmetric photonic crystal by removing a single row of cylinders. The numerical computation of the photonic crystal waveguide modes is important 
(a)

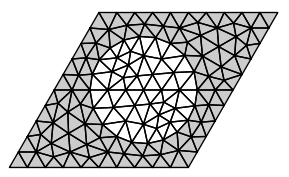

(b)

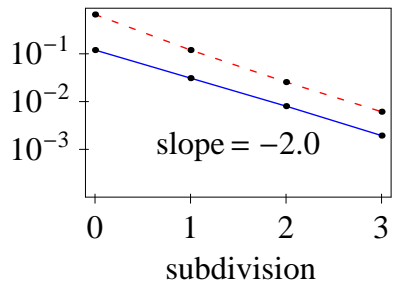

(c)

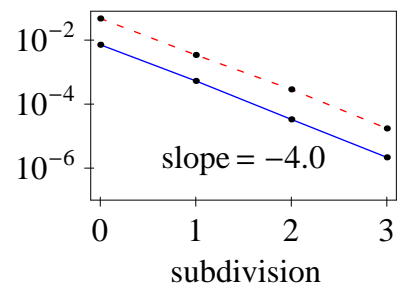

Fig. 3. Convergence of the quadratic FEM. (a) Initial FEM mesh (rectilinear triangles) of the unit cell. The curves on panels (b) and (c) represent the quantities $e(j)=\max _{i}\left\{\left|\mu_{h_{j}}-\mu_{h_{j+1}}\right|\right\}$ as a function of the FEM mesh subdivision $j \in\{0,1,2,3\}$. The continuous and dashed lines indicate respectively the $E$-parallel (TM) and $H$-parallel (TE) modes. The results in (b) are obtained using rectilinear FEM triangles and the rate of convergence is 2. In (c) isoparametric FEM (curved triangles at the cylinder interface) is used and the optimal convergence rate of 4 is achieved.

(a)

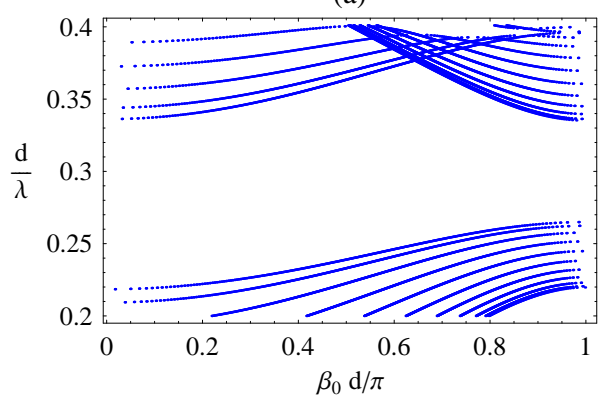

(c)

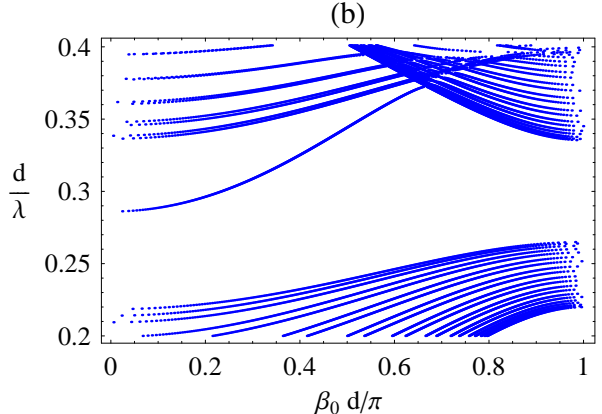

(d)

Fig. 4. (a) Band diagram of a perfect photonic crystal obtained using the grating supercell of length $D=d_{1}=21 d$ shown in the panel (c). (b) Band diagram of a photonic crystal with a line defect; the band is obtained using the supercell of length $D=21 d$ shown in the panel (d). The bands in (a) and (b) are computed along the wave vector line $\mathbf{k}_{\mathbf{0}}=\left(\alpha_{0}, \beta_{0}\right)=\left(0, \beta_{0}\right)$ for the $E$-parallel polarisation.

in many applications and both two and three-dimensional models have been proposed $[24,29]$.

The modelling of a device using the Bloch mode transfer matrix method requires that the defect is embedded within a supercell whose size is chosen to be sufficiently large so that there is negligible crosstalk between adjacent supercells, a consequence of the fields decaying in the cladding due to operation of the device in a band gap. For the method to be practically useful, any calculations should be independent of the Bloch factor $\alpha_{0}$, with the proviso that we operate inside a band gap and that there is effectively no crosstalk between the supercells. This we exemplify with a device referred to as the folded directional coupler (FDC) that we have studied previously [42]. The 
Table 2

The choice of $\alpha_{0}$ has a negligible effect on the computed results: (a) transmittance $T$; (b) propagation constant $\beta$; (grating supercell of length $D=d_{1}=21 d$, wavelength: $\lambda / d=3.3)$.

(a)

\begin{tabular}{lll}
\hline & \multicolumn{2}{c}{$T$} \\
\cline { 2 - 3 }$\frac{D \alpha_{0}}{\pi}$ & $N=20$ & $N=30$ \\
\hline 0. & 0.8207106 & 0.8208297 \\
0.2 & 0.8207106 & 0.8208297 \\
0.4 & 0.8207107 & 0.8208297 \\
0.6 & 0.8207107 & 0.8208296 \\
0.8 & 0.8207105 & 0.8208293 \\
1. & 0.82071 & 0.8208287 \\
\hline
\end{tabular}

(b)

\begin{tabular}{lll}
\hline & \multicolumn{2}{c}{$\beta$} \\
\cline { 2 - 3 }$\frac{D \alpha_{0}}{\pi}$ & $N=20$ & $N=30$ \\
\hline 0. & 0.8111816 & 0.8110291 \\
0.2 & 0.8111817 & 0.8110291 \\
0.4 & 0.8111821 & 0.811029 \\
0.6 & 0.8111829 & 0.811029 \\
0.8 & 0.8111841 & 0.8110291 \\
1. & 0.8111859 & 0.8110295 \\
\hline
\end{tabular}

FDC (Fig. 5 (a)) is a novel high-Q notch rejection filter that exploits the mode coupling properties of a directional coupler and the sharp resonances of a Fabry-Perot (FP) resonator. The structure is made ultra-compact by folding the light path, needed for mode coupling, using a FP interferometer geometry with the aid of cavity mirrors composed of photonic crystal.

The structure that we consider is operated in E-polarised light and comprises a square lattice with cylinders of normalised radius $a / d=0.3$ and refractive index $\nu=3$. Figure 5 (b) depicts the field pattern for a wavelength of $\lambda / d=$ 3.3, while Fig. 5 (c) displays a cross-section of the field intensity at $y=10$ and illustrates the exponential decay of the field in the crystal, with these calculations being performed with $\alpha_{0}=0$. Table 2 (a) displays the variation of the transmittance of the FDC for various values of $\alpha_{0}$ and we see immediately that this is almost independent of $\alpha_{0}$, with the small variation explicable by the small cross-coupling that occurs at the supercell boundaries. A further example of the independence of the calculations on $\alpha_{0}$ is shown in Table 2 (b) which shows the propagation constant $\beta$ for a single waveguide (i.e., the entry or exit guide of the FDC) as a function of $\alpha_{0}$ for a wavelength $\lambda / d=3.3$.

\subsection{Properties of the Bloch modes: the eigenvalue distribution}

We now consider the distribution of eigenvalues in the complex plane for three PCs, respectively having square symmetry, hexagonal symmetry and no defined lattice symmetry. The results are presented in Fig. 6, for which we show only those eigenvalues in the range $10^{-6}<|\mu|<10^{6}$. In order to suitably represent eigenvalues over such a large dynamic range, their magnitude is scaled 
(a)

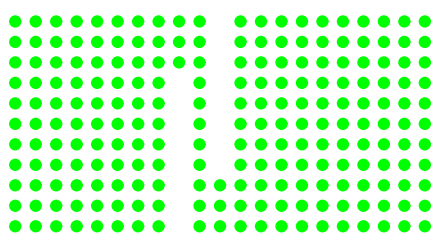

(b)

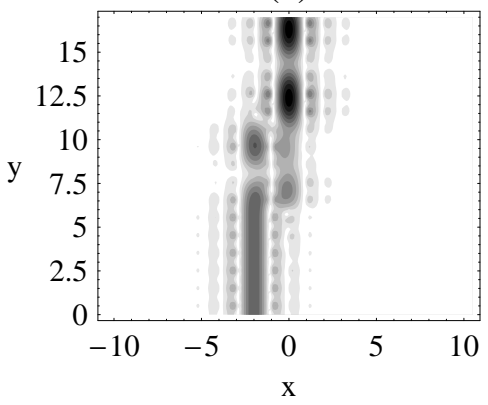

(c)

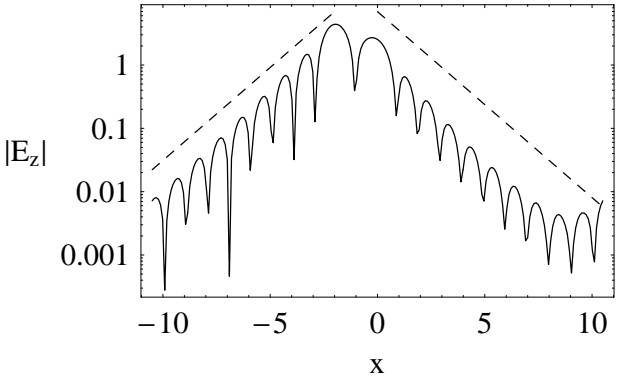

Fig. 5. Folded directional coupler: (a) Geometry; (b) two-dimensional plot of $\left|E_{z}\right|$ at the wavelength $\lambda / d=3.3$; (c) the continuous line represents the cross-section of $\left|E_{z}\right|$ at the level $y=10$ (logarithmic scale); the dashed line represents the function defined by $f(x)=M e^{-\gamma x}$ if $x \geq 0$ and $f(x)=M e^{-\gamma|x+2 d|}$ if $x \leq-2 d$ where $\gamma=-\log |\mu|=0.675973 / d$ is the lowest attenuation constant of the evanescent modes in the bulk photonic crystal (see Fig. 4 (a), (c)) and $M$ is the maximum value of $\left|E_{z}\right|$. The supercell used for the calculations has dimension $d_{1}=21 d$.

radially according to $r=|\mu|^{0.1}$, with the eigenvalues corresponding to propagating states lying on the unit circle. The parameters common to the three problems are as follows: grating period $d_{1}=d$, background refractive index $n_{b}=3$, cylinder refractive index $n_{c}=1$, cylinder radius $a / d=0.3$, free space wavelength $\lambda / d=1$ (TM polarisation), $\alpha_{0}=\pi /(4 d)$.

There are a number of interesting features evident in Fig. 6, one of which is common to all three cases, namely that if $\mu$ is an eigenvalue then $1 / \mu^{*}$ is also an eigenvalue. From this it follows that there is an even number of eigenvalues on the radial lines corresponding to $|\mu| \neq 1$. The proof of this follows from the similarity transformation $\mathcal{T}^{H}=\mathcal{I}_{\mathrm{pw}} \mathcal{T}^{-1} \mathcal{I}_{\mathrm{pw}}^{-1}$ (derived from Eq. (28)) which shows that the eigenvalues $\mu$ and $1 / \mu^{*}$ must be paired.

For the cases (a) and (b) of Fig. 6, the eigenvalue distributions respectively demonstrate axes of symmetry given by the radial directions $\theta=0$ and $\theta=$ $-\alpha_{0} d / 2$. This property can be explained by the fact that the lattices and their corresponding cylinder inclusions are invariant under the transformation $(x, y) \rightarrow(x,-y)$. It is then easy to see that if $v(x, y)$ is a Bloch mode associated with a wave vector $\mathbf{k}_{0}=\left(k_{x}, k_{y}\right)=\left(\alpha_{0}, \beta_{0}\right), \hat{v}(x, y)=v(x,-y)$ is also a Bloch mode associated with the Bloch vector $\hat{\mathbf{k}}_{0}=\left(\alpha_{0},-\beta_{0}\right)$. 
(a)

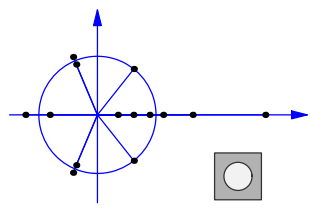

(b)

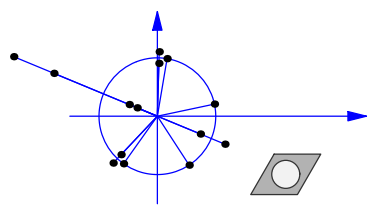

(c)

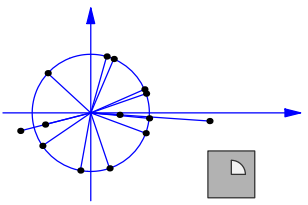

Fig. 6. Eigenvalue distribution (E-parallel polarisation) over the complex number plane. (a) symmetric square lattice PC; (b) symmetric hexagonal lattice PC; (c) nonsymmetric square lattice PC (cylinder with quarter-disk section). The inset shows the unit cell of each case.

For the square lattice we have $\mathbf{e}_{2}=(0, d)$ and so the phase factors corresponding to $\mathbf{k}_{0}$ and $\hat{\mathbf{k}_{0}}$ are respectively $\mu=e^{-i \mathbf{k}_{0} \cdot \mathbf{e}_{2}}=e^{-i \beta_{0} d}$ and $\hat{\mu}=e^{-i \hat{\mathbf{k}_{0}} \cdot \mathbf{e}_{2}}=e^{i \beta_{0} d}$. Thus, for the square symmetric photonic crystal the eigenvalues $\mu$ and $1 / \mu$ are paired and the line $\theta=0$ is an axis of symmetry for the eigenvalues in the complex plane.

For the hexagonal lattice we have $\mathbf{e}_{2}=d(1, \sqrt{3}) / 2$ and so the phase factors corresponding to $\mathbf{k}_{0}$ and $\hat{\mathbf{k}}_{0}$ are respectively $\mu=e^{-i \mathbf{k}_{0} \cdot \mathbf{e}_{2}}=e^{-i\left(\alpha_{0}+\beta_{0} \sqrt{3}\right) d / 2}$ and

$$
\hat{\mu}=e^{-i \hat{\mathbf{k}_{0}} \cdot \mathbf{e}_{2}}=e^{-i\left(\alpha_{0}-\beta_{0} \sqrt{3}\right) d / 2}=e^{-i \alpha_{0} d} e^{i\left(\alpha_{0}+\beta_{0} \sqrt{3}\right) d / 2}=\frac{e^{-i \alpha_{0} d}}{\mu} .
$$

Thus, for the hexagonal lattice the eigenvalues are paired as $\mu e^{i \alpha_{0} d / 2}$ and $1 /\left(\mu e^{i \alpha_{0} d / 2}\right)$ and the line $\theta=-\alpha_{0} d / 2$ is an axis of symmetry for the eigenvalues.

We note that for the case of $\alpha_{0}=0$ the pairing of the eigenvalues $\mu$ and $1 / \mu$ also holds for arbitrary nonsymmetric photonic crystals because of the symplectic nature of the transfer matrix (26), from which the pairing follows from the similarity transformation $\mathcal{T}^{T}=\mathcal{Q}_{\mathrm{pw}} \mathcal{T}^{-1} \mathcal{Q}_{\mathrm{pw}}^{-1}$.

These symmetry properties which are exhibited by the eigenvalue distribution can be used to check the validity and accuracy of the numerical method. The results computed by our FEM satisfy these constraints well. For the eigenvalues appearing on Fig. 6 the pairing $\mu$ and $1 / \mu^{*}$ is verified with a relative error less than $10^{-4} \%$ while the deviation from the axial symmetry of Figs. 6 (a) and (b) is also less than $10^{-4 \%}$.

\subsection{Band structure of a hexagonal lattice of dielectric cylinders}

We next consider the calculation of a band diagram for a hexagonal lattice $\mathrm{PC}$, solving for the dispersion relation $\omega=\omega\left(\mathbf{k}_{0}\right)$ where the wave vector $\mathbf{k}_{0}$ traverses the boundary $\Gamma-K-M$ of the irreducible part of the first Brillouin 
Table 3

Band diagram parameter: $\alpha_{0}=\mathbf{k}_{0} \cdot \mathbf{e}_{1}$ and $\arg (\mu)=\mathbf{k}_{0} \cdot \mathbf{e}_{2}$.

\begin{tabular}{cccccccc}
\hline Path & $\mathbf{k}_{0}$ & $\begin{array}{l}\text { Range } \\
\text { of } s\end{array}$ & $\mathbf{e}_{1} / d$ & $\mathbf{e}_{2} / d$ & $d_{1} / d$ & $\mathbf{k}_{0} \cdot \mathbf{e}_{1}$ & $\begin{array}{c}\text { Range } \\
\text { of } \mathbf{k}_{0} \cdot \mathbf{e}_{2}\end{array}$ \\
\hline$\Gamma-K$ & $s\left(\frac{1}{2}, \frac{\sqrt{3}}{2}\right)$ & {$\left[0, \frac{4 \pi}{3 d}\right]$} & $\left(\frac{3}{2},-\frac{\sqrt{3}}{2}\right)$ & $(1,0)$ & $\sqrt{3}$ & 0 & {$\left[0, \frac{2 \pi}{3}\right]$} \\
$K-M$ & $\left(s, \frac{2 \pi}{d \sqrt{3}}\right)$ & {$\left[0, \frac{2 \pi}{3 d}\right]$} & $(0, \sqrt{3})$ & $\left(\frac{1}{2}, \frac{\sqrt{3}}{2}\right)$ & $\sqrt{3}$ & $2 \pi$ & {$\left[\pi, \frac{4 \pi}{3}\right]$} \\
$M-\Gamma$ & $(0, s)$ & {$\left[0, \frac{2 \pi}{d \sqrt{3}}\right]$} & $(1,0)$ & $\left(\frac{1}{2}, \frac{\sqrt{3}}{2}\right)$ & 1 & 0 & {$[0, \pi]$} \\
$K-M^{\prime}$ & $s\left(\frac{1}{2}, \frac{\sqrt{3}}{2}\right)$ & {$\left[\frac{4 \pi}{3 d}, \frac{2 \pi}{d}\right]$} & $\left(\frac{3}{2},-\frac{\sqrt{3}}{2}\right)$ & $(1,0)$ & $\sqrt{3}$ & 0 & {$\left[\frac{2 \pi}{3}, \pi\right]$} \\
\hline
\end{tabular}

zone shown in Fig. 2 (a). Because of the lattice symmetry, the segment $K-M$ can be replaced by $K-M^{\prime}$ and thus the number of directions to investigate can be reduced to two, namely, $\Gamma-M$ and $\Gamma-K-M^{\prime}$. Since $\mathbf{k}_{0}$ is not a fully independent variable in our numerical algorithm, some care must be taken to ensure that the computed wave vectors belong to a given direction of the reciprocal space.

To obtain the band diagram we use the fact that for any given values of $\alpha_{0}=$ $k_{0 x}$, the wavevectors computed by our method lie on a line perpendicular to the grating layer [6,7]. Wave vectors $\mathbf{k}_{0}$ parallel to the $\Gamma-M$ direction correspond to a normal incidence problem, i.e., $\alpha_{0}=\left(\mathbf{k}_{0} \cdot \mathbf{e}_{1}\right) /\left\|\mathbf{e}_{1}\right\|=0$, on the grating layers shown in Fig. 2 (b) and Fig. 2 (c). Wave vectors $\mathbf{k}_{0}$ parallel to the $\Gamma-K$ direction correspond to a normal incidence problem, i.e., $\alpha_{0}=\left(\mathbf{k}_{0} \cdot \mathbf{e}_{1}\right) /\left\|\mathbf{e}_{1}\right\|=$ 0, on the grating layer shown in Fig. 2 (d). In Table 3, we present the lattice vectors $\mathbf{e}_{1}$ and $\mathbf{e}_{2}$ of Fig. 2 and other parameters used in the computation the band diagram. More details about the procedure can be found in Ref. [7].

The structure of Fig. 2 (b) is straightforward in that the rows of adjacent gratings do not interpenetrate. The grating interior has exactly one row of cylinders and the unit cell has a simple geometry, and so it is relatively easy to generate its finite element mesh. However, when layers interpenetrate, such as in the example of Fig. 2 (c), corresponding to a normalised cylinder radius $a / d>\sqrt{3} / 4=0.433013$, we generate the finite element mesh using the unit cell depicted in Fig. 2 (c). This observation also applies to the case of $\Gamma-K$ incidence, although interpenetration occurs at a far lower normalised radii, $a / d>0.25$. In passing, we observe that the interpenetration of cylinder rows can cause severe difficulties for the multipole method, a problem which is related to the validity of the Rayleigh approximation in diffraction grating theory, and which is discussed in Ref. [7]. In contrast, the FEM approach outlined here has no difficulties in handling such situations.

We now consider two numerical examples: the first a comparison with an FEM based study by Axmann and Kuchment [2], and secondly a comparison with a plane wave calculation for a PC with a high filling fraction taken from Joannopoulos, Meade, and Winn [26]. 
In the Axmann and Kuchment [2] approach, the Bloch vector is set and the frequency becomes the unknown eigenvalue, with the problem being discretised using a linear FEM. This process leads to a generalised eigenvalue problem with a large matrix because it involves directly the values of the unknown fields over the entire unit cell. However this eigenproblem had been efficiently solved using a subspace iteration method that computes only a small number of the physically most relevant (i.e., lowest) eigenvalues. The example considered by Axmann and Kuchment comprised a hexagonal array of dense cylinders of dielectric constant $\varepsilon=14$ and filling fraction 0.431 (i.e., normalised radius $a / d=0.34469)$ embedded in a free space background. The band diagrams in Fig. 7 (a) are very similar to the those of Fig. 2 of Ref. [2].

Table 4 shows the lowest band gaps and there is again good agreement between our isoparametric quadratic finite element technique and the linear FEM presented in Ref. [2]. To obtain results that are stable to 5 significant figures, it is necessary to work with a fine mesh. For the $\Gamma-M$ calculations, the unit cell mesh has 9182 triangles and 18645 points with plane wave orders truncated to lie in the range -22 to 22 , while for the $\Gamma-K-M^{\prime}$ calculations, we used an FEM mesh with 9028 triangles and 18441 points and plane wave orders ranging from -26 to 26 . Note however that the band diagrams in Fig. 7 (a) do not require such a high level of refinement and the curves can be properly obtained using less refined discretisation.

From the results in Fig. 7 (a) and Table 4, it appears that the band gaps for the TE and TM polarizations do not overlap. However Joannopoulos, Meade, and Winn [26] show that, for a hexagonal array of cylinder holes in a high index background, it is possible to achieve a complete band gap for all polarizations if the cylinder radius is large enough. In particular, they considered the case of cylinder holes of normalised radius $a / d=0.48$ in a background with dielectric constant $\varepsilon=13$. Again the band diagram in Fig. 7 (b) shows a good agreement between our FEM and the plane wave method used for the diagram in Ref. [26, p. 65]. The plane wave orders used to obtain the dispersion curves in Fig. 7 (b) range from -19 to 19 and from -23 to 23 respectively for the $\Gamma-M$ and $\Gamma-K-$ $M^{\prime}$ directions. The unit cells are represented by FEM meshes with about 4000 triangles and 8400 points.

\subsection{Band structure of a hexagonal lattice of perfectly conducting metallic cylinders}

We continue on the validation of the method by considering the interesting case of a PC comprising perfectly conducting cylinders which is an excellent approximation for metals in the low frequency limit (e..g., the microwave regime). This problem exhibits an important difference from the dielectric cylinder for- 
Table 4

Comparison of the band gaps (for the normalised frequency $d / \lambda$ in the range $[0,0.5]$ ) obtained using our method (FEM 1) with the results in Table I of [2] (FEM 2).

\begin{tabular}{ccc}
\hline & \multicolumn{2}{c}{ TM } \\
\cline { 2 - 3 } Band gap No & FEM 1 & FEM 2 \\
\hline 1 & {$[0.19644,0.25319]$} & {$[0.19673,0.253637]$} \\
2 & {$[0.34969,0.43569]$} & {$[0.350039,0.436544]$} \\
\hline \multirow{3}{*}{ Band gap No } & TE \\
\cline { 2 - 3 } & FEM 1 & FEM 2 \\
\hline 1 & {$[0.28564,0.33844]$} & {$[0.286089,0.339239]$} \\
\hline
\end{tabular}

(a)

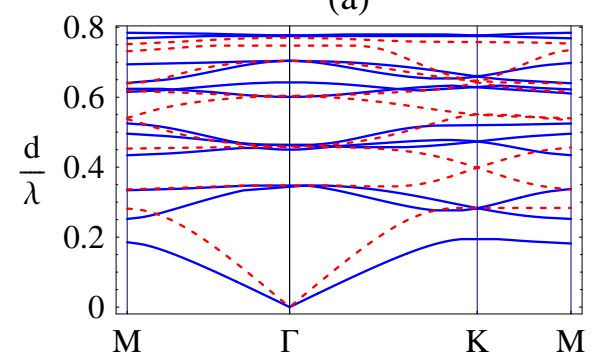

(b)

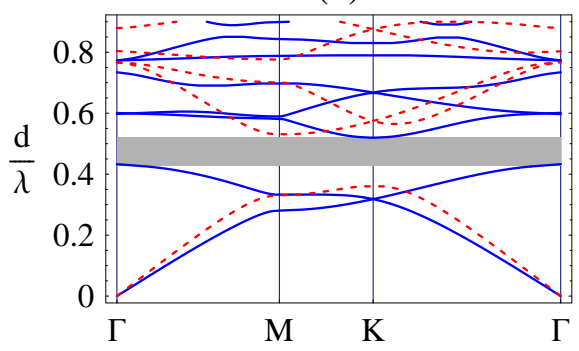

Fig. 7. Band diagram of a hexagonal lattice of cylinders; the continuous and dashed lines indicate respectively the $E$-parallel and $H$-parallel modes. (a) Lattice of cylinder rods of dielectric constant $\varepsilon=14$ and filling fraction 0.431 ; the rods are embedded in a free space background. (b) lattice of cylinder holes of normalised radius $a / d=0.48$ in a background with dielectric constant $\varepsilon=13$

mulation, namely that the interiors of the perfectly conductor cylinders do not belong to the problem domain. Thus complications occur when the grating interfaces $\Pi$ and $\Pi^{\prime}$ intersect these cylinders (as in Figs. 2 (c) and 2 (d)).

In this case, continuity conditions between the plane wave expansion and the field inside the grating have to be enforced on isolated segments of $\Pi$ and $\Pi^{\prime}$. In particular, the second and third equations in (40) are no longer valid because the line integrals on the top and bottom boundaries of the unit cell cover segments of length $L<d_{1}$, and thus the orthogonality of the plane wave functions $\left\{\psi_{m}(x)\right\}$ cannot be applied. This issue can be addressed by replacing $d_{1}$ by $L$ in Eqs. (11) and (40). However the corresponding plane wave representations of the fields are not $d_{1}$-quasi-periodic. Fortunately the $d_{1}$-quasi-periodicity does not have to be directly imposed on the plane wave expansions of the field over the segments of $\Pi$ and $\Pi^{\prime}$ intersecting the grating since these segments are not connected. Accordingly we need only to enforce the $d_{1}$-quasi-periodicity on the field inside the grating. 


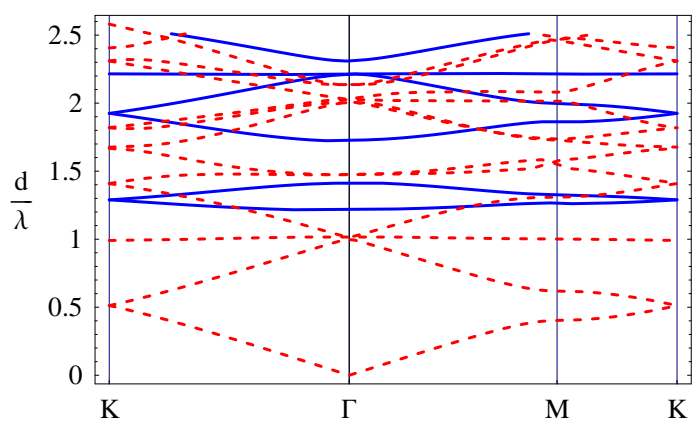

Fig. 8. Band diagram for the perfect metallic cylinder inclusion of radius $a / d=0.34$ in a background medium of refractive index $n_{b}=1$. The continuous and dashed lines indicate respectively the $E$-parallel and $H$-parallel modes.

When the cylinder radius is sufficiently small, we may use either of the two unit cell geometries in Figs. 2 (b) and Fig. 2 (c). Numerical tests show excellent agreement between the band structures obtained by either method. In Fig. 8 we show the band diagram for a hexagonal lattice of perfectly conducting cylindrical inclusions of radius $a / d=0.34$ in a background medium of refractive index $n_{b}=1$. A particular feature of the lattice of metallic cylinders is the existence for $E$-parallel polarisation of a wide band gap from the zero frequency to $d / \lambda=1.211$. The band diagram we obtained is essentially identical to that in Fig. 12 of Ref. [33]. For the calculation of the dispersion curves in Fig. 8, the plane wave orders are truncated to the ranges -14 to 14 and -17 to 17 respectively for the $\Gamma-M$ and $\Gamma-K-M^{\prime}$ directions. The unit cells are represented by FEM meshes with about 2300 triangles and 4900 points.

\subsection{Efficient coupling of light from a photonic crystal waveguide into free space}

Our final example concerns the modelling of photonic crystal waveguides, constructed by removing a single row of cylinders (referred to as a W1 defect waveguide) from an otherwise perfect photonic crystal. Here, we concentrate on the particular question of designing a tapered neck that can be used to apodise, or efficiently couple, light into and out of the waveguide. The aim here is to minimise the reflection that may arise if the transition between the waveguide and free space is not sufficiently smooth.

The determination of an optimal shape for the taper among a set of general, arbitrary shapes can be a very complex problem. Thus to simplify the modelling, the optimisation is typically performed amongst a limited, but physically realistic, set of possible taper profiles. For instance the optimisation of the length and the width of linear taper profiles have be studied in Refs. [36,40]. Here, in addition to the length and the width, as illustrated in Fig. 9, we introduce a third parameter $\xi$ to allow the shape of the taper to be varied continuously 


$$
w(y)=W_{\text {base }}+\left(W-W_{\text {base }}\right)\left(\frac{y}{L d}\right)^{\xi}, \quad \text { for } y \in[0, L d],
$$

where $w(y)$ stands for the width of the taper at the position $y$, the integer $L$ represents the number of grating layers in the taper, $W_{\text {base }} d$ and $W d$ denote respectively the width, defined from the centre of the inclusions, at the lower and upper ends of the taper. For the waveguide of Fig. 9 that we consider, $W_{\text {base }}=2$. The positive parameter $\xi$ determines the taper shape: for $\xi=1$ the taper is linear, for $\xi>1$ it is concave as in Fig. 9, while for $\xi<1$ it is convex.

The square lattice photonic crystal structure illustrated in Fig. 9 comprises three regions: the waveguide, the taper region (shaded rectangle in Fig. 9) and free space, a semi-infinite homogeneous medium having the same refractive index as the photonic crystal background material.

Each constituent layer of the taper or the characteristic layer of the waveguide is treated as a diffraction grating having a supercell period of length $D=21 d$ which we operate with a periodic boundary condition $\left(\alpha_{0}=0\right)$. Inside each of the grating layers that comprise the taper, the cylinders on the taper wall are placed so that the centre-to-centre separation is given by Eq. (57). As indicated in Fig. 9, the distance between the two inclusions closest to the taper opening is maintained at $d$, whereas subsequent inclusions are separated by $d^{\prime}$, such that $d^{\prime}$ is as close as possible to $d$, consistent with the supercell period $D ; d^{\prime}$ generally differs in each of the gratings. Near the taper opening, we choose to maintain a cylinder separation of $d$, since it is important to maintain the integrity of the band gap that is responsible for confining the light in the vicinity of the taper core. The problem we will consider is to determine the optimal taper profile for efficient transmission from the fundamental mode of the photonic crystal waveguide into free space. To perform the analysis we will need to compute the reflected field in the waveguide and the transmitted field in free space for a sequence of taper profiles corresponding to incidence of the fundamental waveguide mode. Within the waveguide, we represent the field in terms of a Bloch mode basis obtained from the solution of the eigenvalue problem for the waveguide mode transfer matrix (23). The scattering property of the taper is then characterised in terms of its reflection and transmission matrices. Details of the algorithm are given in Appendix A.

We consider a rod-type photonic crystal in $E$-polarised light (i.e., TM polarisation), consisting of cylinders of radius $a=0.25 d$, and refractive index $n_{c}=3$ arranged in a square array, in a background of refractive index $n_{b}=1$. Each layer of the taper is represented by a finite element mesh of around 12000 triangles and 25000 points, with the plane wave expansion truncated to include orders ranging from -29 to 29 . The bulk photonic crystal has a band gap 


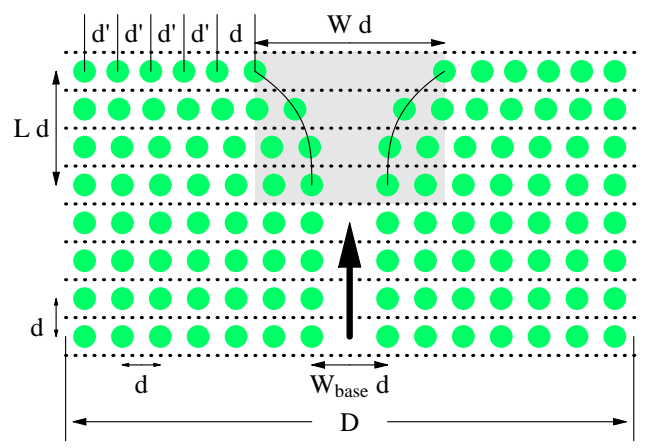

Fig. 9. Schematic illustration of the square lattice taper. Light is incident from at he fundamental mode of a photonic crystal waveguide (large arrow) into free space via a taper of $L$ layers and width $W d$ (shaded rectangle). The photonic crystal is considered to consist of a sequence of gratings (horizontal dotted lines) with supercell period $D$. The solid curves correspond to Eq. 57 specifying the positions of the scatterers.

for normalised frequencies $d / \lambda \in[0.287,0.387]$, i.e., for wavelengths $\lambda$ lying between $2.582 d$ and $3.487 d$. Within this gap, the W1 waveguide supports a mode with a cutoff at $d / \lambda=0.302$, i.e., $\lambda=3.312 d$, so the relevant wavelength range for our study of coupling from the waveguide into free space is $\lambda / d \in[2.582,3.312]$.

The transmittance of the fundamental mode over this wavelength region is shown in Fig. 10 for tapers of total width $W=4$, with length ranging from $L=4$ to $L=8$ and for various taper parameters $\xi$. The contour plots of Fig. 10 have levels ranging from very high values of $99.5 \%$ down to values of $70 \%$ reflecting poor taper performance. In general, waveguide tapers with $2 \leq \xi \leq 3$ (horn-shaped tapers) are capable of delivering very high transmittances. This is also evident in Fig. 10(b) which shows the transmission spectrum for the optimal taper, and for a waveguide that is terminated abruptly (i.e., without any tapering). For tapers of this optimal shape, the transmittance and wavelength coverage increase with taper length, with a length of six periods delivering transmittances not significantly below those of longer tapers. Note that from Fig. 10 we see that linear tapers $(\xi=1)$ can deliver good performance. However, to achieve this the taper has to be longer than for a taper of optimal shape.

Also, in Fig. 10(c) we plot the group velocity. In the case of an infinite waveguide, we may calculate this from $v_{g}=d \omega / d \beta$ of from the the ratio of the energy flux in the guide to the energy density in a layer [7]. For a semi-infinite structure, such as a terminated waveguide, however, the group velocity can be computed only with the second method, in which we must estimate the energy density by averaging over a number of layers deep in the waveguide in order to avoid any truncation effects which manifest themselves in the form of evanescent waveguide modes which are prominent near the interface. The 
dashed curve in Fig. 10(c) is the group velocity in the optimal taper, with the energy density being averaged over layers 11 layers some 10 layers below the start of the taper. Coinciding perfectly with this (although invisible on the graph) is the group velocity of the infinite guide, computed according to $v_{g}=d \omega / d \beta$, with the perfect agreement of the two associated with the optimal tapering. The solid curve in Fig. 10(c) is the group velocity for the untapered termination, and we see that this differs markedly from the group velocity for the optimal structure. In both cases, however, we see that the transmittance is qualitatively similar to the group velocity, with both vanishing at the cutoff wavelength. However, the relationship between transmittance and group velocity is somewhat indirect, since transmittance is primarily a function of impedance mismatch at an interface while the group velocity is essentially a characteristic of the mode dispersion properties.

In many of the examples we have studied [16], involving different waveguide geometries (square and hexagonal lattices), waveguide length and polarisation, optimal transmittance tends to be delivered by tapers with $2 \leq \xi \leq 3$. In Ref. [16], we have also studied hexagonal lattices, the modelling for which is significantly more complex since the rows of closely packed cylinders can interpenetrate. It is here that the accuracy and flexibility of the FEM implementation of the Bloch mode tools in handling challenging structures is particularly useful.

\section{Conclusion}

In this paper we have proposed a numerical method, based on a scattering matrix formalism, for the analysis of two-dimensional photonic crystals. A finite element method for the computation of the scattering matrices has been presented. Since the most computationally expensive step, associated with the factorisation of FEM matrices, is not repeated, the construction of scattering matrices is carried out quite efficiently. The mode structure can then be obtained from the scattering matrices by solving low dimension and numerically stable eigenvalue problems. The scattering matrix formalism can also be used to analyse light propagation through finite size structures. The method is accurate, computationally robust and can be applied to arbitrary periodic materials, including lossy media. Extensions of the approach to 3D structure are possible and will be pursued in the future. 
(a)
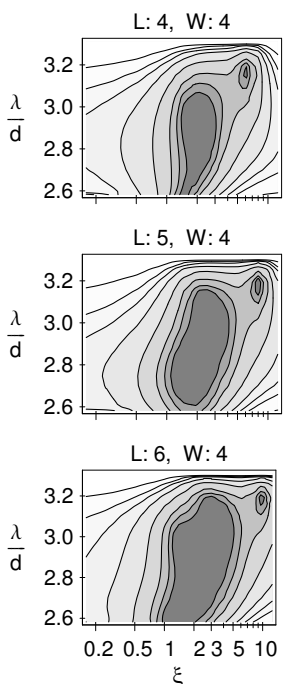

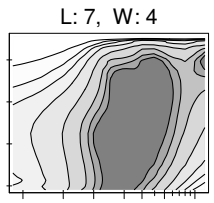

L: 8, W: 4
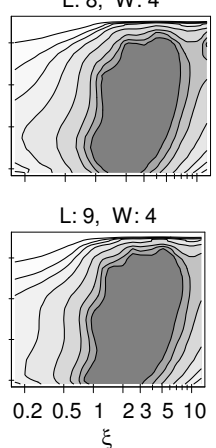

(b)

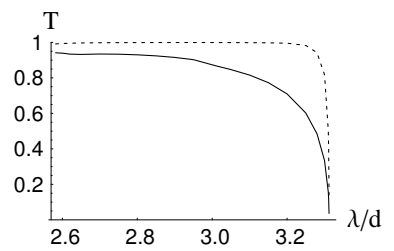

(c)

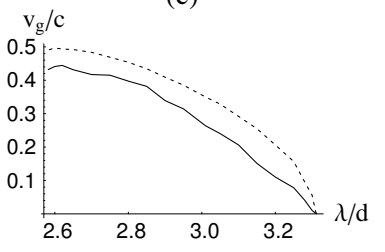

Fig. 10. (a) Transmittance from a square lattice photonic crystal waveguide into free space for a taper of width $W=4$ and lengths $L=4,5, \ldots, 9$ layers. The contour levels shown correspond to transmittances of 0.995, 0.99, 0.98, 0.95, 0.90, $0.85,0.80$ and 0.70 , decreasing from the region centred on $\xi=2$ outwards. (b) The continuous and dashed lines represent respectively the transmittance into free space for a waveguide without a taper and for a waveguide having a taper with parameter $L=7, W=4$ and $\xi=3$. (c) Group velocity $v_{g} / c$ ( $c$ is the free space speed of light) of the two structures considered in (b).

\section{Acknowledgement}

This work was produced with the assistance of the Australian Research Council under the ARC Centres of Excellence Program.

\section{Appendix A: efficient coupling from photonic crystal waveguides into free space}

In this appendix we give details of the algorithm used in Sec. 5.5 to compute the reflectance and transmittance of a tapered PC waveguide (see Fig. 9).

To solve the propagation problem for a waveguide mode incident from below the taper, we need the Fresnel reflection $\left(\mathbf{R}_{\text {taper }}^{\prime}\right)$ and transmission $\left(\mathbf{T}_{\text {taper }}^{\prime}\right)$ matrices of the taper. To obtain these matrices we first compute the scattering matrices $\mathbf{R}_{l}, \mathbf{T}_{l}, \mathbf{R}_{l}^{\prime}, \mathbf{T}_{l}^{\prime}$ for the taper layers $l=1,2, \ldots, L$, enumerating these from bottom to top. Note that from the up-down symmetry of the layers, we have $\mathbf{R}_{l}^{\prime}=\mathbf{R}_{l}$ and $\mathbf{T}_{l}^{\prime}=\mathbf{T}_{l}$, and thus the calculation of the scattering 
matrices can be halved. We will append the subscripted notation $l_{1}, l_{2}$ to the scattering matrices variables to denote the reflection or transmission of a stack of layers ranging from the layer $l_{1}$ to layer $l_{2}$; for instance $\mathbf{R}_{\text {taper }}^{\prime}=\mathbf{R}_{1, L}^{\prime}$ and $\mathbf{T}_{\text {taper }}^{\prime}=\mathbf{T}_{1, L}^{\prime}$. We then form the reflection and transmission matrices for the taper associated with incidence from below through backward recursion

$$
\begin{aligned}
& \mathbf{R}_{l-1, L}^{\prime}=\mathbf{R}_{l-1}^{\prime}+\mathbf{T}_{l-1} \mathbf{R}_{l, L}^{\prime}\left(\mathbf{I}-\mathbf{R}_{l-1} \mathbf{R}_{l, L}^{\prime}\right)^{-1} \mathbf{T}_{l-1}^{\prime} \\
& \mathbf{T}_{l-1, L}^{\prime}=\mathbf{T}_{l, L}^{\prime}\left(\mathbf{I}-\mathbf{R}_{l-1} \mathbf{R}_{l, L}^{\prime}\right)^{-1} \mathbf{T}_{l-1}^{\prime}
\end{aligned}
$$

commencing with $\mathbf{R}_{L, L}^{\prime}=\mathbf{R}_{L}^{\prime}$ and $\mathbf{T}_{L, L}^{\prime}=\mathbf{T}_{L}^{\prime}$.

In the waveguide region, the fields are expanded in the Bloch mode basis, obtained from the diagonalisation of the waveguide transfer matrix (23). If a field of upward propagating modes (designated by a vector of Bloch mode coefficients $\mathbf{c}_{+}$) is incident on the interface between the waveguide and the taper, it will generate a reflected field of downward propagating modes $\left(\mathbf{c}_{-}\right)$. Thus, at the lower interface of the taper, the field is expressed in terms of a plane wave expansion (13) with upward and downward components $\left(\mathbf{g}_{ \pm}\right)$given by

$$
\left[\begin{array}{l}
\mathbf{g}_{-} \\
\mathbf{g}_{+}
\end{array}\right]=\left[\begin{array}{l}
\mathbf{F}_{-} \\
\mathbf{F}_{+}
\end{array}\right] \mathbf{c}_{-}+\left[\begin{array}{l}
\mathbf{F}_{-}^{\prime} \\
\mathbf{F}_{+}^{\prime}
\end{array}\right] \mathbf{c}_{+}=\left[\begin{array}{l}
\mathbf{F}_{-} \mathbf{F}_{-}^{\prime} \\
\mathbf{F}_{+} \mathbf{F}_{+}^{\prime}
\end{array}\right]\left[\begin{array}{l}
\mathbf{c}_{-} \\
\mathbf{c}_{+}
\end{array}\right]
$$

The interaction of the plane wave fields $\mathbf{g}_{ \pm}$and the field $\mathbf{t}$ transmitted into free space is characterised by the taper reflection and transmission matrices defined by

$$
\mathbf{g}_{-}=\mathbf{R}_{\text {taper }}^{\prime} \mathbf{g}_{+}, \quad \mathbf{t}=\mathbf{T}_{\text {taper }}^{\prime} \mathbf{g}_{+}
$$

Solving Eqs. (60) and (61), we arrive at expressions for the reflection $\left(\mathbf{R}_{\mathrm{gf}}\right)$ and transmission $\left(\mathbf{T}_{\mathrm{gf}}\right)$ matrices of the "guide-taper-free space" system, defined by

$$
\mathbf{c}_{-}=\mathbf{R}_{\mathrm{gf}} \mathbf{c}_{+}, \quad \mathbf{t}=\mathbf{T}_{\mathrm{gf}} \mathbf{c}_{+} .
$$

We thus derive

$$
\begin{aligned}
& \mathbf{R}_{\mathrm{gf}}=\left(\mathbf{F}_{-}\right)^{-1}\left(\mathbf{I}-\mathbf{R}_{\text {taper }}^{\prime} \mathbf{R}_{g}\right)^{-1}\left(\mathbf{R}_{\text {taper }}^{\prime}-\mathbf{R}_{g}^{\prime}\right) \mathbf{F}_{+}^{\prime}, \\
& \mathbf{T}_{\mathrm{gf}}=\mathbf{T}_{\text {taper }}^{\prime}\left(\mathbf{I}-\mathbf{R}_{g} \mathbf{R}_{\text {taper }}^{\prime}\right)^{-1}\left(\mathbf{I}-\mathbf{R}_{g} \mathbf{R}_{g}^{\prime}\right) \mathbf{F}_{+}^{\prime} .
\end{aligned}
$$


The quantities $\mathbf{R}_{g}=\mathbf{F}_{+}\left(\mathbf{F}_{-}\right)^{-1}$ and $\mathbf{R}_{g}^{\prime}=\mathbf{F}_{-}^{\prime}\left(\mathbf{F}_{+}^{\prime}\right)^{-1}$ that appear in each of Eqs. (63) and (64) are the plane wave reflection matrices of semi-infinite photonic crystals, respectively corresponding to incidence from above and below $[7,10]$.

For a specified incident modal field $\mathbf{c}_{+}$, the reflected modal field $\mathbf{c}_{-}$and the transmitted plane wave field $\mathbf{t}$ may then be computed using Eq. (62). If the plane wave and waveguide basis are normalised so that each mode carries unit energy, we can express the energy conservation of the system as follows

$$
\sum_{m \in \Omega_{g}}\left|c_{m}^{(-)}\right|^{2}=\sum_{m \in \Omega_{g}}\left|c_{m}^{(+)}\right|^{2}+\sum_{p \in \Omega_{f}}\left|t_{p}\right|^{2}
$$

provided that the incident waveguide field contains only propagating modal terms [10]. In Eq. (65), $\Omega_{m}$ and $\Omega_{f}$ respectively denote the set of propagating waveguide modes and the set of propagating plane waves.

\section{References}

[1] N. W. Ashcroft and N. D. Mermin. Solid state physics. Holt, Rinehart and Winston, New York, 1976.

[2] W. Axmann and P. Kuchment. An efficient finite element method for computing spectra of photonic and acoustic band-gap materials. I. Scalar case. J. Comput. Phys., 150(2):468-481, 1999.

[3] G. Bao and D. C. Dobson. Variational methods for diffractive optics modeling. In Mathematical modeling in optical science, volume 22 of Frontiers Appl. Math., pages 37-69. SIAM, Philadelphia, PA, 2001.

[4] S. Boscolo, M. Midrio, and T. F. Krauss. Y junctions in photonic crystal channel waveguides: high transmission and impedance matching. Opt. Lett., 27(12):1001-1003, 2002.

[5] S. Boscolo, M. Midrio, and C.G. Someda. Coupling and decoupling of electromagnetic waves in parallel $2 \mathrm{D}$ photonic crystal waveguides. IEEE $J$. Quantum Electron., 38(1):47-53, 2002.

[6] L. C. Botten, R. C. McPhedran, C. M. de Sterke, N. A. Nicorovici, A. A. Asatryan, G. H. Smith, T. N. Langtry, T. P. White, D. P. Fussell, and B. T. Kuhlmey. From multipole methods to photonic crystal device modelling. In K. Yasumoto, editor, Electromagnetic Theory and Applications for Photonic Crystals, chapter 2. CRC Press, 2005.

[7] L. C. Botten, N. A. Nicorovici, R. C. McPhedran, C. M. de Sterke, and A. A. Asatryan. Photonic band structure calculations using scattering matrices. Phys. Rev. E, 64:046603, 2001. 
[8] L. C. Botten, N.-A. P. Nicorovici, A. A. Asatryan, R. C. McPhedran, C. M. de Sterke, and P. A. Robinson. Formulation for electromagnetic scattering and propagation through grating stacks of metallic and dielectric cylinders for photonic crystal calculations. Part I. Method. J. Opt. Soc. Am. A, 17(12):2165$2176,2000$.

[9] L. C. Botten, N.-A. P. Nicorovici, A. A. Asatryan, R. C. McPhedran, C. M. de Sterke, and P. A. Robinson. Formulation for electromagnetic scattering and propagation through grating stacks of metallic and dielectric cylinders for photonic crystal calculations. Part II. Properties and implementation. J. Opt. Soc. Am. A, 17(12):2177-2190, 2000.

[10] L. C. Botten, T. P. White, A. A. Asatryan, T. N. Langtry, C. M. de Sterke, and R. C. McPhedran. Bloch mode scattering matrix methods for modeling extended photonic crystal structures. I. Theory. Phys. Rev. E, 70:056606, 2004.

[11] Q. Cao, P. Lalanne, and J.-P. Hugonin. Stable and efficient Bloch-mode computational method for one-dimensional grating waveguides. J. Opt. Soc. Am. A, 19(2):335-338, 2002.

[12] P. G. Ciarlet. The finite element method for elliptic problems. Society for Industrial and Applied Mathematics (SIAM), Philadelphia, USA, 2002. Reprint of the 1978 original [North-Holland, Amsterdam].

[13] A. Delâge and K. Dossou. Polarisation dependent loss calculation in echelle gratings using finite element method and Rayleigh expansion. Opt. Quant. Electron., 36(1-3):223-238, 2004.

[14] T. Delort and D. Maystre. Finite-element method for gratings. J. Opt. Soc. Am. A, 10(12):2592-2601, 1993.

[15] D. C. Dobson. An efficient method for band structure calculations in 2D photonic crystals. J. Comput. Phys., 149(2):363-376, 1999.

[16] K. Dossou, L. C. Botten, S. Chen, J. Brnovic, C. M. de Sterke, R. C. McPhedran, and A. A. Asatryan. Efficient couplers for photonic crystal waveguides. to appear in the journal Optics Communications, 2005.

[17] K. Dossou, M. Packirisamy, and M. Fontaine. Analysis of diffraction gratings by using an edge element method. J. Opt. Soc. Am. A, 22(2):278-288, 2005.

[18] M. S. P. Eastham. The spectral theory of periodic differential equations. Scottish Academic Press, Edinburgh, 1973.

[19] J. Elschner and G. Schmidt. Diffraction in periodic structures and optimal design of binary gratings. I. Direct problems and gradient formulas. Math. Methods Appl. Sci., 21(14):1297-1342, 1998.

[20] S. Fan, S. G. Johnson, J. D. Joannopoulos, C. Manolatou, and H. A. Haus. Waveguide branches in photonic crystals. J. Opt. Soc. Am. B, 18(2):162-165, 2001. 
[21] S. Fan, P. R. Villeneuve, J. D. Joannopoulos, and H. A. Haus. Channel drop tunneling through localized states. Phys. Rev. Lett., 80(5):960-963, 1998.

[22] Gmsh. http://www.geuz.org/gmsh/.

[23] B. Gralak, S. Enoch, and G. Tayeb. From scattering or impedance matrices to Bloch modes of photonic crystals. J. Opt. Soc. Am. A, 19(8):1547-1554, 2002.

[24] G.R. Hadley. Out-of-plane losses of line-defect photonic crystal waveguides. IEEE Photon. Technol. Lett., 14(5):2002, 642-644.

[25] S. F. Helfert. Determination of floquet modes in asymmetric periodic structures, optical and quantum electronics. Opt. Quant. Electron., 37(1-3):185-197, 2005.

[26] J. D. Joannopoulos, R. D. Meade, and J. N. Winn. Photonic Crystals: Molding the Flow of Light. Princeton University Press, 1995.

[27] S. G. Johnson and J. D. Joannopoulos. Block-iterative frequency-domain methods for Maxwell's equations in a planewave basis. Opt. Express, 8:173190, 2001.

[28] H. Kosaka, T. Kawashima, A. Tomita, M. Notomi, T. Tamamura, T. Sato, and S. Kawakami. Photonic crystals for micro lightwave circuits using wavelengthdependent angular beam steering. Appl. Phys. Lett., 74(10):1370-1372, 1999.

[29] P. Lalanne. Electromagnetic analysis of photonic crystal waveguides operating above the light cone. IEEE J. Quantum Electron., 38(7):800-804, 2002.

[30] LAPACK. http://www.netlib.org/lapack/.

[31] Z.-Y. Li and L.-L. Lin. Photonic band structures solved by a plane-wave-based transfer-matrix method. Phys. Rev. E, 67:046607, 2003.

[32] A. Martinez, A. Griol, P. Sanchis, and J. Marti. Mach Zehnder interferometer employing coupled-resonator optical waveguides. Opt. Lett., 28(6):405-407, 2003.

[33] N. A. Nicorovici, R. C. McPhedran, and L. C. Botten. Photonic band gaps for arrays of perfectly conducting cylinders. Phys. Rev. E, 52:1135-1145, 1995.

[34] J. B. Pendry and A. MacKinnon. Calculation of photon dispersion relations. Phys. Rev. Lett., 69:2772-2775, 1992.

[35] R. Petit, editor. Electromagnetic theory of gratings, volume 22 of Topics in Current Physics. Springer-Verlag, New York, 1980.

[36] P. Pottier, I. Ntakis, and R. M. De La Rue. Photonic crystal continuous taper for low-loss direct coupling into 2D photonic crystal channel waveguides and further device functionality. Opt. Comm., 223(4-6):339-347, 2003.

[37] M. Reed and B. Simon. Methods of modern mathematical physics, Vol. IV: Analysis of operators. Academic Press, New York, 1978.

[38] K. Sakoda. Optical Properties of Photonic Crystals. Springer, New York, second edition, 2005. 
[39] N. Stefanou, V. Karathanos, and A. Modinos. Scattering of electromagnetic waves by periodic structures. J. Phys.: Condens. Matter, 4(36):7389-7400, 1992.

[40] A. Talneau, M. Agio, C. M. Soukoulis, M. Mulot, S. Anand, and Lalanne Ph. High-bandwidth transmission of an efficient photonic-crystal mode converter. Opt. Lett., 29(15):1745-1747, 2004.

[41] A. Talneau, L. Le Gouezigou, N. Bouadma, M. Kafesaki, C. M. Soukoulis, and M. Agio. Photonic-crystal ultrashort bends with improved transmission and low reflection at $1.55 \mu \mathrm{m}$. Appl. Phys. Lett., 80(4):547-549, 2002.

[42] T. P. White, L. C. Botten, R. C. McPhedran, and C. M. de Sterke. Ultracompact resonant filters in photonic crystals. Opt. Lett., 28(24):2452-2454, 2003.

[43] S. Wolfram. The Mathematica book. Wolfram Media / Cambridge University Press, 3rd edition, 1996. 